\title{
Limited-area atmospheric energetics: illustration on a simulation of the CRCM5 over eastern North America for December 2004
}

\author{
Marilys Clément ${ }^{1} \cdot$ Oumarou Nikiéma $^{1} \cdot$ René Laprise $^{1}$
}

Received: 16 October 2015 / Accepted: 20 May 2016 / Published online: 3 June 2016

(c) The Author(s) 2016. This article is published with open access at Springerlink.com

\begin{abstract}
The seminal work of Lorenz on global atmospheric energetics has allowed understanding much on the physical processes responsible for the general circulation, its maintenance and the development of synoptic-scale weather systems. In mid-latitudes, potential energy generated by the differential heating of the planet by the Sun is converted into kinetic energy by the weather systems and eventually dissipated by friction. While a corresponding study of atmospheric energetics over a limited region would have the advantage of focusing on the details of individual storms and the processes taking place over a domain of interest, such study has encountered several pitfalls and has been challenging. Here we build upon our earlier work on the energy cycle of inter-member variability in ensembles of limited-area model simulations to develop a regionalscale atmospheric energy cycle formulated in terms of available enthalpy and kinetic energy. The approach is then applied to study the energetics of a short simulation made with the fifth-generation of the Canadian Regional Climate Model (CRCM5) over an eastern North American domain for December 2004. The results obtained for a specific storm and monthly mean climatology confirm the current understanding that available enthalpy of the atmospheric time-mean state is mainly generated by the covariance of diabatic heating and temperature, and that the most important conversions of energy are found to correspond to baroclinic processes that take place along the storm track, where perturbations of temperature and wind are important.
\end{abstract}

Marilys Clément

marilys.clement@gmail.com

1 Département des sciences de la Terre et de l'atmosphère, Centre ESCER (Étude et Simulation du Climat à l'Échelle Régionale), UQÀM, Stn. Downtown, P.O. Box 8888, Montréal, QC H3C 3P8, Canada
Finally, transient-eddy kinetic energy is mainly dissipated by friction in the boundary layer.

Keywords Regional atmospheric energy budget - Limitedarea domain $\cdot$ Regional Climate Model $\cdot$ North American climate

\section{List of symbols}

$a_{T V} \quad$ Instantaneous available enthalpy for time variability

$c_{a} \quad$ Instantaneous conversion of time-mean into time variability available enthalpy

$c_{k} \quad$ Instantaneous conversion of time-mean into time variability kinetic energy

$c_{p} \quad$ Specific heat at constant pressure for dry air

$c_{T V} \quad$ Instantaneous conversion of time variability available enthalpy into time variability kinetic energy

$d_{T V} \quad$ Instantaneous dissipation of time variability available enthalpy

$f_{a_{T V}} \quad$ Instantaneous transport of time variability available enthalpy

$f_{k_{T V}} \quad$ Instantaneous transport of time variability kinetic energy

$g_{T V} \quad$ Instantaneous generation of time variability available enthalpy

$h_{a_{T V}} \quad$ Instantaneous boundary flux of time variability available enthalpy

$h_{k_{T V}} \quad$ Instantaneous boundary flux of time variability kinetic energy

$j_{a 1} \quad$ Available enthalpy flux 1 for instantaneous energy cycle

$j_{a 2} \quad$ Available enthalpy flux 2 for instantaneous energy cycle

$j_{k 1} \quad$ Kinetic energy flux 1 for instantaneous energy cycle 


\begin{tabular}{|c|c|c|c|}
\hline$k_{T V}$ & $\begin{array}{l}\text { Instantaneous available enthalpy for time } \\
\text { variability }\end{array}$ & $H_{A_{T M B}}$ & $\begin{array}{l}\text { Boundary flux of time-mean baroclinic avail- } \\
\text { able enthalpy }\end{array}$ \\
\hline$l$ & Factor of order unity & \multirow[t]{2}{*}{$H_{A_{T M S}}$} & Boundary flux of time-mean stratification avail- \\
\hline$p$ & Pressure & & able enthalpy \\
\hline$p_{00}$ & Domain average of surface pressure & \multirow[t]{2}{*}{$H_{A_{T V}}$} & \multirow{2}{*}{$\begin{array}{l}\text { Boundary flux of time variability available } \\
\text { enthalpy }\end{array}$} \\
\hline$p_{r}$ & Reference pressure & & \\
\hline$t$ & Number of time samples used for computation & $H_{K_{T M}}$ & Boundary flux of time-mean kinetic energy \\
\hline \multirow[t]{2}{*}{$A$} & \multirow{2}{*}{$\begin{array}{l}\text { Temperature-dependent part of available } \\
\text { enthalpy }\end{array}$} & \multirow{4}{*}{$\begin{array}{l}H_{K_{T V}} \\
I_{A B}\end{array}$} & Boundary flux of time variability kinetic energy \\
\hline & & & Conversion of pressure-dependent available \\
\hline$A E$ & Marquet's available enthalpy & & enthalpy into time-mean stratification available \\
\hline$A P E$ & Available potential energy & & enthalpy \\
\hline$A_{T M}$ & Available enthalpy for time-mean state & $I E$ & Internal energy \\
\hline \multirow[t]{2}{*}{$A_{T M B}$} & \multirow{2}{*}{$\begin{array}{l}\text { Baroclinic component of available enthalpy for } \\
\text { time-mean state }\end{array}$} & IV & Inter-member variability \\
\hline & & $K$ & Kinetic energy \\
\hline \multirow[t]{2}{*}{$A_{T M S}$} & \multirow{2}{*}{$\begin{array}{l}\text { Stratification component of available enthalpy } \\
\text { for time-mean state }\end{array}$} & $K_{T M}$ & Kinetic energy for time-mean state \\
\hline & & $K_{T V}$ & Kinetic energy for time variability \\
\hline$A_{T V}$ & Available enthalpy for time variability & $K E$ & Margules' kinetic energy \\
\hline$B$ & Pressure-dependent part of available enthalpy & $L_{E}$ & Time-tendency of energy reservoir $E$ \\
\hline \multirow[t]{2}{*}{$C_{A B}$} & \multirow{2}{*}{$\begin{array}{l}\text { Conversion of time-mean baroclinic available } \\
\text { enthalpy into time variability available enthalpy }\end{array}$} & $P E$ & Margules’ potential energy \\
\hline & & $R$ & Gas constant for air \\
\hline$C_{A S}$ & $\begin{array}{l}\text { Conversion of time-mean stratification available } \\
\text { enthalpy into time variability available enthalpy }\end{array}$ & $R_{E}$ & $\begin{array}{l}\text { Algebraic sum of all fluxes acting upon energy } \\
\text { reservoir } E\end{array}$ \\
\hline \multirow[t]{2}{*}{$C_{K}$} & \multirow{2}{*}{$\begin{array}{l}\text { Conversion of time-mean into time variability } \\
\text { kinetic energy }\end{array}$} & $\mathrm{RCM}$ & Regional Climate Model \\
\hline & & $T$ & Temperature \\
\hline \multirow[t]{2}{*}{$C_{T M B}$} & \multirow{2}{*}{$\begin{array}{l}\text { Conversion of time-mean baroclinic available } \\
\text { enthalpy into time-mean kinetic energy }\end{array}$} & $T_{r}$ & Reference temperature \\
\hline & & $T P E$ & Lorenz' total potential energy \\
\hline \multirow[t]{3}{*}{$C_{T M B S}$} & \multirow{3}{*}{$\begin{array}{l}\text { Conversion of time-mean baroclinic available } \\
\text { enthalpy into time-mean stratification available } \\
\text { enthalpy }\end{array}$} & $U P E$ & Lorenz' unavailable potential energy \\
\hline & & $\vec{V}$ & Horizontal wind \\
\hline & & $\alpha$ & Specific volume \\
\hline \multirow[t]{2}{*}{$C_{T M S}$} & \multirow{2}{*}{$\begin{array}{l}\text { Conversion of time-mean stratification available } \\
\text { enthalpy into time-mean kinetic energy }\end{array}$} & $\alpha_{r}$ & Reference specific volume \\
\hline & & $\tau$ & Number of time samples \\
\hline \multirow[t]{2}{*}{$C_{T V}$} & \multirow{2}{*}{$\begin{array}{l}\text { Conversion of time variability available } \\
\text { enthalpy into time variability kinetic energy }\end{array}$} & $\omega$ & Vertical velocity in pressure coordinates \\
\hline & & $\Phi$ & Geopotential height \\
\hline \multirow[t]{2}{*}{ CRCM5 } & \multirow{2}{*}{$\begin{array}{l}\text { Fifth-generation of the Canadian Regional Cli- } \\
\text { mate Model }\end{array}$} & $\Psi$ & General atmospheric variable \\
\hline & & $\langle\Psi\rangle$ & Time-mean operator \\
\hline$D_{T M}$ & Dissipation of time-mean kinetic energy & $\Psi^{\prime}$ & Time deviation operator \\
\hline$D_{T V}$ & Dissipation of time variability kinetic energy & $\bar{\Psi}$ & Isobaric mean operator \\
\hline$E$ & General type of atmospheric energy & $\Psi^{\times}$ & Isobaric deviation operator \\
\hline
\end{tabular}

\section{Introduction}

The study of atmospheric energetics provides fundamental informations characterising the physical behaviour and maintenance of the general circulation. Since the 1950's, many computations of atmospheric energy cycle have been made on the global scale using data from atmospheric analyses or from simulations of global climate models (GCM). These studies have provided detailed insights on the transformations and conversions of various forms of energy in the atmosphere.

Margules (1905; see also Tamura 1905) was one of the first to study the atmospheric energetics. He recognized 
the different forms of energy present in the atmosphere as being the kinetic energy $(K E)$ associated with the horizontal wind, the gravitational potential energy $(P E)$ due to the gravitational attraction by the Earth, and the internal energy (IE) caused by the Brownian motion of the molecules. He noted that $I E$ and $P E$, when integrated over a column of the atmosphere in hydrostatic equilibrium, are proportional to one another and hence he introduced the total potential energy (TPE) defined as the sum of $I E$ and $P E$. He faced however practical difficulties in calculating quantitative energy budgets due to TPE being much larger than $K E$, and the fluxes of TPE being huge but their convergence modest.

Lorenz $(1955,1967)$ was the first to develop a detailed tractable atmospheric energy cycle and his work is still today considered as a classic in atmospheric energetics. He introduced the concept of available potential energy ( $A P E$ ). $T P E$ is separated into unavailable potential energy (UPE), the TPE of a reference state, and APE, the excess of TPE over that reference state. The reference state is defined as that which would exist if the total mass of the atmosphere were redistributed adiabatically, resulting in a horizontal density stratification everywhere. In such a notional barotropic atmosphere, UPE could not be transformed into $K E$ because there would be no pressure gradient to generate wind. Consequently, it is only the much smaller $A P E$ component of TPE that can be transformed into KE. Lorenz therefore developed an energy cycle based on $A P E$ and $K E$ as the only two relevant forms of energy in the atmosphere. To better understand the role of weather systems in the atmospheric energetics, Lorenz further decomposed the energy fields into components associated with the zonalmean atmospheric state and departures thereof, termed eddies. Much of our current understanding of global atmospheric energetics derives from Lorenz' seminal work (e.g. Oort 1964a, b; Newell et al. 1972, 1974; Boer 1974; Pexioto and Oort 1992; to name just a few).

There are however noteworthy limitations associated with Lorenz' approach. An important one is that the concepts of TPE and APE are only meaningful when applied to the entire atmosphere, not for a portion of it (van Mieghem 1973, Sect. 14.8). Several mathematical manipulations used to establish APE and associated conversion terms require integrating over the entire atmosphere, causing several terms to vanish identically. There are further approximations introduced to simplify the definition of the basic state to compute UPE and to cope with topography. Hence, computing the energetics of a limited region is much more challenging (e.g. Spar 1950; Smith 1969a, b). First, a reference minimum-energy state needs to be defined without a global framework. Second, domain boundary fluxes need to be considered, and hence flux-divergence terms that would cancel when integrated over the whole atmosphere need to be retained. And third, the domain mass is not conserved and the domain-averaged vertical velocity does not vanish, generating additional terms in the energy budget.

Still, understanding the physical processes responsible for the formation and development of individual weather systems, and the underlying energy conversions, remains a long-standing scientific interest. The recent advent of highresolution atmospheric analyses and regional model simulations facilitates the access to the required data and stimulate such undertaking.

Pearce (1978) developed a distinct energy cycle approach by redefining $A P E$ as the departure from an isothermal reference state. His $A P E$ is therefore a direct function of the variance of the temperature, which facilitates its calculation and physical interpretation. Similarly to Lorenz, Pearce decomposed his fields of $A P E$ and $K E$ into zonalmean and eddy components. He also decomposed $A P E$ into a function of the vertical distribution of temperature, accounting for changes in static stability. As a result, his definition of $A P E$ is easier to interpret physically and better adapted to regional diagnostics in the atmosphere. Following Pearce's approach, Marquet (2003a, b) established an exact local and complete energy cycle applicable for a limited-area domain. His formulation is based on available enthalpy $(A E)$, which naturally separates into contributions from temperature and pressure, allowing this cycle to be used on any pressure level if desired.

Recently, Nikiéma and Laprise (2013, noted "NL13" hereafter), used an approximate form of this formalism to develop an energy budget of inter-member variability (IV) in ensemble simulations of a nested Regional Climate Model (RCM), decomposing atmospheric fields into ensemble-mean and inter-member departures.

NL13 noted that a close parallel could be drawn between the IV energy conversions and the transient eddy energy conversions taking place in weather systems. Therefore, the purpose of this paper is first to formulate a limited-area energy budget of transient eddies following closely the approach employed by NL13 for IV, and second, to apply this energy cycle to study the energetics of weather systems in an RCM simulation over an eastern North American domain. Available enthalpy $(A E)$ and kinetic energy (hereafter $K$ ) will be decomposed in components relating to the time-mean state of the atmosphere and departures thereof, the transient eddy or time variability. The paper is organised as follows. Section 2 will develop the formalism for the energy budget. Section 3 will describe the experimental design and characterize the climate of the studied period. Sections 4 and 5 will present the results and analysis of the energetics of a specific storm and of the monthly-mean energetics of December 2004, respectively. Finally, Sect. 6 will summarise the findings and conclude. 


\section{Methodology for the computation of an atmospheric energy cycle}

Following closely the mathematical development of NL13 for the energetics of IV in RCM ensemble simulations, an atmospheric energy cycle applicable over a limited-area domain is here derived. The main difference is that atmospheric variables $\Psi \in\{T, \vec{V}, \Phi, \ldots\}$, instead of being decomposed into their ensemble-mean state and intermember deviations as in NL13, are here decomposed into their time-mean state $\langle\Psi\rangle=\frac{1}{\tau} \sum_{t=1}^{\tau} \Psi_{t}$ with $\tau$ the number of time samples, and time deviations $\Psi^{\prime}=\Psi-\langle\Psi\rangle$, so that $\Psi=\langle\Psi\rangle+\Psi^{\prime}$. Furthermore, the mathematical development has been extended to allow the computation of instantaneous time evolution of energetics, rendering the energy cycle applicable to the study of specific storms.

The available enthalpy $A E$ is decomposed into its pressure-dependent component $B$ and temperature-dependent component $A$ (Marquet 1991, used in NL13). The component $A$ is further decomposed into components associated with the time-mean state of the atmosphere $A_{T M} \propto\langle T\rangle^{2}$ and its time deviations, the instantaneous time-variability state $a_{T V} \propto T^{\prime 2}$, with its time average $A_{T V} \equiv\left\langle a_{T V}\right\rangle \propto\left\langle T^{\prime 2}\right\rangle$. The kinetic energy is also decomposed into a component associated with the time-mean state of the atmosphere $K_{T M} \propto\langle\vec{V}\rangle^{2}$ and its time deviations, the instantaneous time variability state $k_{T V} \propto \vec{V}^{\prime 2}$, with its time average $K_{T V}=\left\langle k_{T V}\right\rangle \propto\left\langle\vec{V}^{\prime 2}\right\rangle$. The use of lower and upper cases serves to differentiate energies associated with instantaneous time deviation of variables in lower case from their time-averaged statistics in upper case. The detailed equations for each reservoir are presented in the two following Subsections.

\subsection{Storm energy cycle equations}

For the study of the life cycle of a storm, only the instantaneous time variability energy reservoirs $a_{T V}$ and $k_{T V}$ need to be analysed; the time-mean energy reservoirs not being of much interest given that they are not notably affected during the passage of a single storm. The details of the development for the $a_{T V}$ and $k_{T V}$ equations are provided in

"Appendix 1: development for $\partial a_{T V} / \partial t$ " and "Appendix 2: development for $\partial k_{T V} / \partial t$ ", respectively.

$a_{T V}$ is the instantaneous available enthalpy associated with the time fluctuations of temperature. Its equation is:

$\frac{\partial a_{T V}}{\partial t}=g_{T V}-c_{T V}+c_{a}-f_{a_{T V}}-h_{a_{T V}}-j_{a 1}-j_{a 2}$

where

$a_{T V}=\frac{c_{p}}{2 T_{r}}\left(T^{\prime}\right)^{2}$
$g_{T V}=l\left(\frac{T^{\prime}}{T_{r}} Q^{\prime}\right)$ with $l$ a factor of order unity

$c_{T V}=-\omega^{\prime} \alpha^{\prime}$

$c_{a}=-\left(\overrightarrow{V^{\prime}} \frac{c_{p} T^{\prime}}{T_{r}}\right) \cdot \vec{\nabla}\langle T\rangle-\left(\omega^{\prime} \frac{c_{p} T^{\prime}}{T_{r}}\right) \frac{\partial\langle T\rangle}{\partial p}$

$f_{a_{T V}}=\vec{\nabla} \cdot\left(a_{T V}\langle\vec{V}\rangle\right)+\frac{\partial\left(a_{T V}\langle\omega\rangle\right)}{\partial p}$

$h_{a_{T V}}=\left(\vec{\nabla} \cdot\left(a_{T V} \overrightarrow{V^{\prime}}\right)+\frac{\partial\left(a_{T V} \omega^{\prime}\right)}{\partial p}\right)$

$j_{a 1}=-\frac{c_{p} T^{\prime}}{T_{r}} \vec{\nabla} \cdot\left\langle\vec{\nabla}^{\prime} T^{\prime}\right\rangle-\frac{c_{p} T^{\prime}}{T_{r}} \frac{\partial\left\langle\omega^{\prime} T^{\prime}\right\rangle}{\partial p}, \quad j_{a 2}=-T^{\prime}\left\langle\frac{Q}{T}\right\rangle$

$k_{T V}$ is the instantaneous kinetic energy associated with the time fluctuations of horizontal wind. Its equation is:

$\frac{\partial k_{T V}}{\partial t}=c_{T V}+c_{k}-d_{T V}-f_{k_{T V}}-h_{k_{T V}}-j_{k 1}$

where

$k_{T V}=\frac{1}{2} \overrightarrow{V^{\prime}} \cdot \overrightarrow{V^{\prime}}$

$c_{T V}=-\omega^{\prime} \alpha^{\prime}$

$c_{k}=-\overrightarrow{V^{\prime}} \cdot\left(\overrightarrow{V^{\prime}} \cdot \vec{V}\right)\langle\vec{V}\rangle-\overrightarrow{V^{\prime}} \cdot\left(\omega^{\prime} \frac{\partial\langle\vec{V}\rangle}{\partial p}\right)$

$d_{T V}=-\overrightarrow{V^{\prime}} \cdot \overrightarrow{F^{\prime}}$

$f_{k_{T V}}=\vec{\nabla} \cdot\left(k_{T V}\langle\vec{V}\rangle\right)+\frac{\partial\left(k_{T V}\langle\omega\rangle\right)}{\partial p}$

$h_{k_{T V}}=\frac{1}{2} \vec{\nabla} \cdot\left(\left(\overrightarrow{V^{\prime}} \cdot \overrightarrow{V^{\prime}}+\Phi^{\prime}\right) \overrightarrow{V^{\prime}}\right)+\frac{1}{2} \frac{\partial\left(\left(\overrightarrow{V^{\prime}} \cdot \overrightarrow{V^{\prime}}+\Phi^{\prime}\right) \omega\right)}{\partial p}$

$j_{k 1}=-\overrightarrow{V^{\prime}}\left\langle\overrightarrow{V^{\prime}} \cdot \vec{\nabla} \overrightarrow{V^{\prime}}\right\rangle-\overrightarrow{V^{\prime}}\left\langle\omega^{\prime} \frac{\partial \overrightarrow{V^{\prime}}}{\partial p}\right\rangle$

Equation $(1,2)$ constitute an approximate set of prognostic equations describing the instantaneous exchanges of energy between the time variability available enthalpy and kinetic energy, suitable for the study of a storm's energetics over a regional domain. The equations reveal that the 


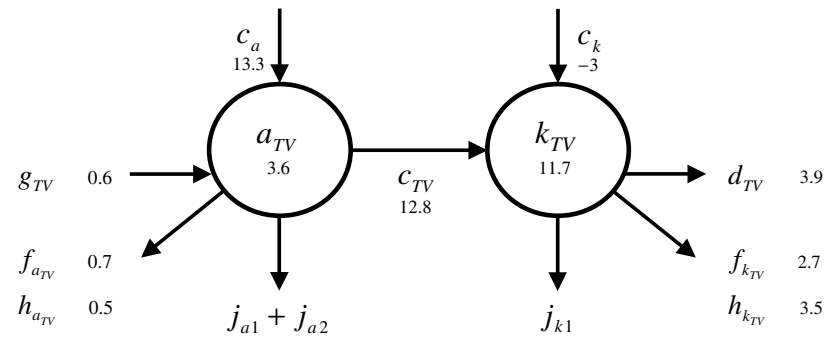

Fig. 1 Vertically integrated time- and domain-averaged storm energy cycle. Values are computed for the duration of the storm (December 26 to 29, 2004. Energy reservoirs (boxes) are shown in $10^{5} \mathrm{~J} \mathrm{~m}^{-2}$ and energy fluxes (arrows) in $\mathrm{W} \mathrm{m}^{-2}$

energy reservoirs $a_{T V}$ and $k_{T V}$ are linked together, resulting in the instantaneous energy cycle illustrated in Fig. 1, where boxes represent energy reservoirs and arrows indicate energy exchanges between reservoirs and with the environment. The direction of the arrows is arbitrary and solely reflects the choice of sign used for defining the terms in the equations. An arrow pointing toward (away from) a reservoir indicates that the energy exchange corresponds to a source (sink) of energy for that reservoir, but only the calculations will reveal whether its value is positive or negative. The magnitude and interpretation of all terms will be discussed in Sect. 4.

The terms $c$ correspond to conversions of energy between different reservoirs. The term $g$ generates available enthalpy; when it corresponds to a positive (negative) covariance of perturbations of temperature and diabatic heating, it represents a source (sink) of available enthalpy. The term $d$ corresponds to dissipation of kinetic energy due to friction and vertical diffusion. The friction is mainly present in the boundary layer, with maximum values where the surface stress is largest: over rough terrain and where the wind is the strongest. The terms $f$ are boundary fluxes representing the transport of energy by the time-mean wind. Averaged over the domain of interest, these terms act as sinks (sources) of energy if more (less) energy leaves the domain than enters it, and they would cancel out for an energy cycle computed over the whole globe. The terms $h$ are also boundary fluxes of energy that would cancel out for an energy cycle computed over the whole globe. The terms $j$ are third-order terms.

\subsection{Energy cycle climatology equations}

For the energy cycle climatology, both the time-mean and the time-averaged variability energy reservoirs are studied. In the following analysis, the prognostic equations for the five energy reservoirs $A_{T M}, A_{T V}, K_{T M}, K_{T V}$ and $B$ will only be considered for their horizontally averaged values on isobaric surfaces. The horizontal average on isobaric surfaces is noted $\bar{\Psi}$ and its deviation $\Psi^{\times}=\Psi-\bar{\Psi}$.
$A_{T M}$ is the available enthalpy associated with the timemean temperature. It represents the departure of the time-averaged temperature from a reference temperature $T_{r}=260 \mathrm{~K}$. Following Marquet (1991) the reference temperature is chosen so that $1 / T_{r}=\overline{\langle 1 / T\rangle}$, with $\overline{\langle\rangle}$ the time and domain average over the period and domain of interest. The isobaric average causes the reservoir $A_{T M}$ to split into two contributions: $\overline{A_{T M}}=\overline{A_{T M S}}+\overline{A_{T M B}}$ (see NL13's Sect. 6). This separation provides a better representation of available enthalpy and is physically meaningful, justifying this undertaking. The set of six approximate energy equations developed by NL13 (Appendix 11) is here presented for an atmospheric energy cycle averaged on isobaric surfaces, valid for a limited-area domain.

$A_{T M S}$ is the part of $A_{T M}$ due to the mean stratification of the atmosphere. It is the largest component of $A_{T M}$ and its vertical structure reflects the specific choice of $T_{r}$, as in Nikiéma et al. (2016) for IV energy cycle. The equation for $A_{T M S}$ is:

$\frac{\partial \overline{A_{T M S}}}{\partial t}=\overline{G_{T M S}}+\overline{I_{A B}}-\overline{C_{T M S}}-\overline{C_{T M B S}}-\overline{C_{A S}}-\overline{F_{A_{T M S}}}-\overline{H_{A_{T M S}}}$

where

$$
\begin{aligned}
& \overline{A_{T M S}}=\frac{c_{p}}{2 T_{r}}\left\langle\bar{T}-T_{r}\right\rangle^{2} \\
& \overline{G_{T M S}}=\frac{l}{T_{r}}\left(\left(\overline{\langle T\rangle}-T_{r}\right) \overline{\langle Q\rangle}\right) \\
& \overline{I_{A B}}=-\frac{R T_{r}}{p} \overline{\langle\omega\rangle} \\
& \overline{C_{T M S}}=-\overline{\langle\omega\rangle}\left(\overline{\langle\alpha\rangle}-\alpha_{r}\right) \\
& \overline{C_{T M B S}}=-\frac{c_{p}}{T_{r}} \overline{\left\langle\frac{\partial \bar{T}}{\partial p} \omega^{\times}\right\rangle\left\langle T^{\times}\right\rangle} \\
& \overline{C_{A S}}=-\frac{c_{p}}{T_{r}}\left(\overline{\left\langle\overrightarrow{V^{\prime}} \overline{T^{\prime}}\right\rangle \cdot \vec{\nabla}\langle T\rangle}\right)-\frac{c_{p}}{T_{r}}\left(\overline{\left\langle\omega^{\prime} T^{\prime}\right\rangle} \frac{\partial \overline{\langle T\rangle}}{\partial p}+\overline{\left.\left\langle\omega^{\prime} \overline{T^{\prime}}\right\rangle \frac{\partial\left\langle T^{\times}\right\rangle}{\partial p}\right)}\right.
\end{aligned}
$$$$
\overline{F_{A_{T M S}}}=\vec{\nabla} \cdot\left(\overline{A_{T M S}\langle\vec{V}\rangle}\right)+\frac{\partial\left(\overline{A_{T M S}\langle\omega\rangle}\right)}{\partial p}
$$$$
\overline{H_{A_{T M S}}}=\frac{c_{p}}{T_{r}}\left(\vec{\nabla} \cdot\left(\overline{\left\langle T_{*}\right\rangle} \overline{\left\langle\overrightarrow{V^{\prime}} T^{\prime}\right\rangle}+\overline{\left\langle T^{\times}\right\rangle\left\langle\overrightarrow{V^{\prime}} \overline{T^{\prime}}\right\rangle}\right)\right)
$$$$
+\frac{c_{p}}{T_{r}}\left(\frac{\partial\left(\overline{\left\langle T_{*}\right\rangle\left\langle\omega^{\prime} T^{\prime}\right\rangle}+\overline{\left\langle T^{\times}\right\rangle\left\langle\omega^{\overline{T^{\prime}}}\right\rangle}\right)}{\partial p}\right)
$$ 
$A_{T M B}$ is the part of $A_{T M}$ due to the baroclinicity of the atmosphere. It generally has smaller values than $A_{T M S}$ and is of the same order of magnitude as $K_{T M}$, as in Nikiéma and Laprise (2016) for IV energy cycle. The value of $A_{T M} B$ decreases with height and the maximum energy is near the ground where the temperature deviation with respect to the isobaric mean temperature is the largest. The equation for $A_{T M} B$ is:

$\frac{\partial \overline{A_{T M B}}}{\partial t}=\overline{G_{T M B}}-\overline{C_{T M B}}+\overline{C_{T M B S}}-\overline{C_{A B}}-\overline{F_{A_{T M B}}}-\overline{H_{A_{T M B}}}$

where

$$
\begin{aligned}
& \overline{A_{T M B}}=\frac{c_{p}}{2 T_{r}} \overline{\left\langle T^{\times}\right\rangle^{2}} \\
& \overline{G_{T M B}}=\frac{l}{T_{r}} \overline{\left\langle T^{\times}\right\rangle\left\langle Q^{\times}\right\rangle} \\
& \overline{C_{T M B}}=-\overline{\left\langle\omega^{\times}\right\rangle\left\langle\alpha^{\times}\right\rangle} \\
& \overline{C_{T M B S}}=-\frac{c_{p}}{T_{r}} \overline{\left\langle\frac{\partial \bar{T}}{\partial p} \omega^{\times}\right\rangle\left\langle T^{\times}\right\rangle}
\end{aligned}
$$$$
\overline{C_{A B}}=-\frac{c_{p}}{T_{r}}\left(\overline{\left\langle\overrightarrow{V^{\prime}} T^{\prime \times}\right\rangle \cdot \vec{\nabla}\langle T\rangle}\right)-\frac{c_{p}}{T_{r}}\left(\overline{\left\langle\omega^{\prime} T^{\prime \times}\right\rangle \frac{\partial\left\langle T^{\times}\right\rangle}{\partial p}}\right)
$$$$
\overline{F_{A_{T M B}}}=\vec{\nabla} \cdot\left(\overline{A_{T M B}\langle\vec{V}\rangle}\right)+\frac{\partial\left(\overline{A_{T M S}\langle\omega\rangle}\right)}{\partial p}
$$$$
\overline{H_{A_{T M B}}}=\frac{c_{p}}{T_{r}} \vec{\nabla} \cdot\left(\overline{\left\langle T^{\times}\right\rangle\left\langle{\overrightarrow{V^{\prime}}}^{\prime \prime}\right\rangle}\right)+\frac{c_{p}}{T_{r}}\left(\frac{\partial\left(\overline{\left\langle T^{\times}\right\rangle\left\langle\omega^{\prime \times}\right\rangle}\right)}{\partial p}\right)
$$

$A_{T V}$ is the available enthalpy associated with the time variability of temperature. It corresponds to the time-average of $a_{T V}$ defined earlier, averaged on isobaric surfaces. The equation for $A_{T V}$ is:

$\frac{\partial \overline{A_{T V}}}{\partial t}=\overline{G_{T V}}-\overline{C_{T V}}+\overline{C_{A S}}+\overline{C_{A B}}-\overline{F_{A_{T V}}}-\overline{H_{A_{T V}}}$

Where

$$
\begin{aligned}
& \overline{A_{T V}}=\frac{c_{p}}{2 T_{r}}\left\langle T^{\prime 2}\right\rangle \\
& \overline{C_{T V}}=\frac{l}{T_{r}}\left\langle\overline{T^{\prime} Q^{\prime}}\right\rangle \\
& \overline{C_{T V}}=\left\langle\overline{\omega^{\prime} \alpha^{\prime}}\right\rangle
\end{aligned}
$$

$\overline{C_{A B}}=\frac{c_{p}}{T_{r}}\left(\overline{\left\langle\overrightarrow{V^{\prime}} T^{\prime \times}\right\rangle \cdot \vec{\nabla}\langle T\rangle}\right)-\frac{c_{p}}{T_{r}}\left(\overline{\left\langle\omega^{\prime} T^{\prime \times}\right\rangle \cdot \frac{\partial\left\langle T^{\times}\right\rangle}{\partial p}}\right)$

$\overline{C_{A S}}=\frac{c_{p}}{T_{r}}\left(\overline{\left\langle\overrightarrow{V^{\prime}} \overline{T^{\prime}}\right\rangle \cdot \vec{\nabla}\langle T\rangle}\right)-\frac{c_{p}}{T_{r}}\left(\left\langle\overline{\omega^{\prime} T^{\prime \times}}\right\rangle \cdot \frac{\partial \overline{\langle T\rangle}}{\partial p}+\overline{\left\langle\omega^{\prime} \overline{T^{\prime \times}}\right\rangle \cdot \overline{\frac{\partial\left\langle T^{\times}\right\rangle}{\partial p}}}\right)$

$\overline{F_{A_{T V}}}=\vec{\nabla}\left(\overline{A_{T V}\langle\vec{V}\rangle}\right)+\frac{\partial\left(\overline{A_{T V}\langle\omega\rangle}\right)}{\partial p}$

$\overline{H_{A_{T V}}}=\frac{c_{p}}{2 T_{r}}\left\langle\vec{\nabla} \cdot\left(\overline{\overrightarrow{\nabla^{\prime}} T^{\prime 2}}\right)+\frac{\partial\left(\overline{\omega^{\prime} T^{\prime 2}}\right)}{\partial p}\right\rangle$

$K_{T M}$ is the kinetic energy associated with the time-mean wind. Its equation is:

$\frac{\partial \overline{K_{T M}}}{\partial t}=\overline{C_{T M} S}+\overline{C_{T M} B}-\overline{C_{K}}-\overline{D_{T M}}-\overline{F_{K_{T M}}}-\overline{H_{K_{T M}}}$

where

$$
\begin{aligned}
& \overline{K_{T M}}=\frac{1}{2} \overline{\langle\vec{V}\rangle \cdot\langle\vec{V}\rangle} \\
& \overline{C_{T M S}}=-\overline{\langle\omega\rangle}\left(\overline{\langle\alpha\rangle}-\alpha_{r}\right) \\
& \overline{C_{T M B}}=-\overline{\left\langle\omega^{\times}\right\rangle\left\langle\alpha^{\times}\right\rangle} \\
& \overline{C_{K}}=-\overline{\left\langle\overrightarrow{V^{\prime}} \cdot\left(\overrightarrow{V^{\prime}} \cdot \vec{\nabla}\right)\langle\vec{V}\rangle\right\rangle}-\overline{\left\langle\overrightarrow{V^{\prime}} \cdot\left(\omega^{\prime} \frac{\partial\langle\vec{V}\rangle}{\partial p}\right)\right\rangle} \\
& \overline{D_{T M}}=-\overline{\langle\vec{V}\rangle \cdot\langle\vec{F}\rangle}
\end{aligned}
$$

$\overline{F_{K_{T M}}}=\vec{\nabla} \cdot\left(\overline{K_{T M}\langle\vec{V}\rangle}\right)+\frac{\partial\left(\overline{K_{T M}\langle\omega\rangle}\right)}{\partial p}$

$$
\begin{aligned}
\overline{H_{K_{T M}}}= & \vec{\nabla} \cdot\left(\overline{\langle\vec{V}\rangle \cdot\left\langle\overrightarrow{\left.V^{\prime} \vec{V}^{\prime}\right\rangle}\right)}+\vec{\nabla} \cdot(\overline{\langle\vec{V}\rangle\langle\Phi\rangle})\right. \\
& +\frac{\partial\left(\overline{\langle\vec{V}\rangle \cdot\left\langle\overrightarrow{\left.V^{\prime} \omega^{\prime}\right\rangle}\right.}\right)}{\partial p}+\frac{\partial(\overline{\langle\omega\rangle\langle\Phi\rangle})}{\partial p}
\end{aligned}
$$

$K_{T V}$ is the kinetic energy associated with the time variability of the wind. It corresponds to the time-average of $k_{T V}$ defined earlier, averaged on isobaric surfaces. The equation for $K_{T V}$ is: 
Fig. 2 Vertically integrated time- and domain-averaged energy cycle climatology. Energy reservoirs (boxes) are shown in $10^{5} \mathrm{~J} \mathrm{~m}^{-2}$ and energy fluxes (arrows) in $\mathrm{W} \mathrm{m}^{-2}$

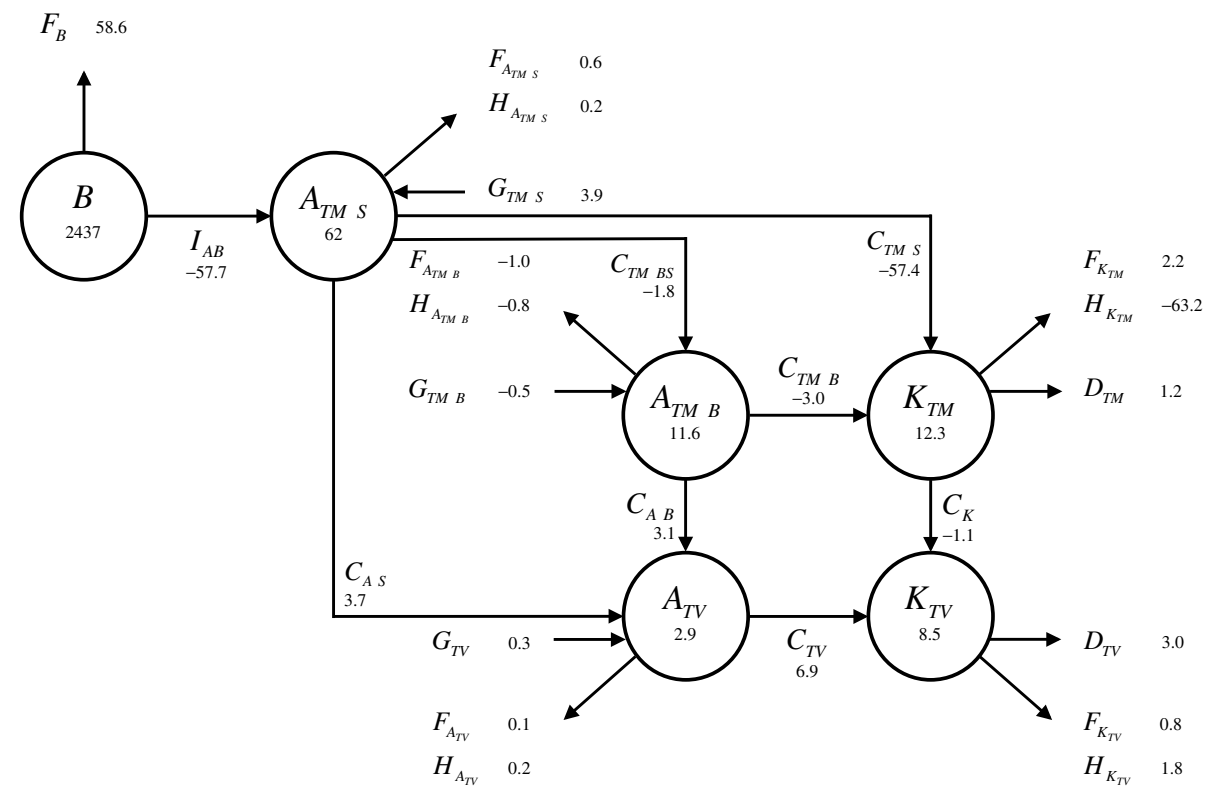

surface pressure. As the surface pressure does not vary a lot in relative terms, $B$ is very close to being constant in time. The equation for $B$ is:

$\frac{\partial \bar{B}}{\partial t}=-\overline{F_{B}}-\overline{I_{A B}}$

$\overline{K_{T V}}=\frac{1}{2} \overline{\left\langle\overrightarrow{V^{\prime}} \cdot \overrightarrow{V^{\prime}}\right\rangle}$

where

$\overline{C_{T V}}=-\overline{\left\langle\omega^{\prime} \alpha^{\prime}\right\rangle}$

$\overline{C_{K}}=\overline{\left\langle\overrightarrow{V^{\prime}} \cdot\left(\overrightarrow{V^{\prime}} \cdot \vec{\nabla}\right)\langle\vec{V}\rangle\right\rangle}-\overline{\left\langle\overrightarrow{V^{\prime}} \cdot\left(\omega^{\prime} \frac{\partial\langle\vec{V}\rangle}{\partial p}\right)\right\rangle}$

$\overline{D_{T V}}=-\overline{\left\langle\overrightarrow{V^{\prime}} \cdot \overrightarrow{F^{\prime}}\right\rangle}$

$\overline{F_{A_{T V}}}=\vec{\nabla} \cdot\left(\overline{K_{T V}\langle\vec{V}\rangle}\right)+\frac{\partial\left(\overline{K_{T V}\langle\omega\rangle}\right)}{\partial p}$

$\overline{H_{K_{T V}}}=\vec{\nabla} \cdot \overline{\left\langle\left(\frac{1}{2} \overrightarrow{V^{\prime}} \overrightarrow{V^{\prime}}+\Phi^{\prime}\right) \overrightarrow{V^{\prime}}\right\rangle}+\frac{\partial \overline{\left\langle\left(\frac{1}{2} \overrightarrow{V^{\prime}} \overrightarrow{V^{\prime}}+\Phi^{\prime}\right) \omega^{\prime}\right\rangle}}{\partial p}$

$B$ is the pressure-dependent part of available enthalpy and its variations reflect the fact that the mass is not constant over a limited-area domain. It represents the effect on $A E$ of the departure from a reference pressure $p_{r} \simeq p_{00} / e$, with the constant $p_{00}$ approximately the mean value of the
$\overline{F_{B}}=\vec{\nabla} \cdot(\overline{B\langle\vec{V}\rangle})+\frac{\partial(\overline{B\langle\omega\rangle})}{\partial p}$

$\bar{B}=R T_{r} \overline{\left\langle\ln \frac{p}{p_{r}}\right\rangle}$

$\overline{I_{A B}}=-\frac{R T_{r}}{p} \overline{\langle\omega\rangle}$

Equations (3-8) constitute an approximate set of equations describing the exchanges of energy between the timemean and time variability states of available enthalpy and kinetic energy, appropriate for the study of climatological energetics over a regional domain. There are six energy reservoirs corresponding to the six prognostic equations of $\overline{A_{T M S}}, \overline{A_{T M B}}, \overline{A_{T V}}, \overline{K_{T M}}, \overline{K_{T V}}$ and $\bar{B}$. The set of equations is linked together resulting in the energy cycle illustrated in Fig. 2, where again, boxes and arrows represent energy reservoirs and energy exchanges. The magnitude and interpretation of the terms will be discussed in Sect. 5 .

The terms $I$ and $C$ are conversion of energy between different reservoirs; they convert energy types from available enthalpy to kinetic energy and energy states from timemean to time variability. The terms $G$ represent generation 
Fig. 3 Maps of a terrain elevation $(\mathrm{m})$, b vertically averaged time-mean temperature (K), c vertically averaged time-mean wind $\left(\mathrm{m} \mathrm{s}^{-1}\right)$, and $\mathbf{d}$ time-averaged mean sea level pressure (hPa) with semi-permanent high- and low-pressure systems indicated. The time-average is taken for the month of December 2004 (a)

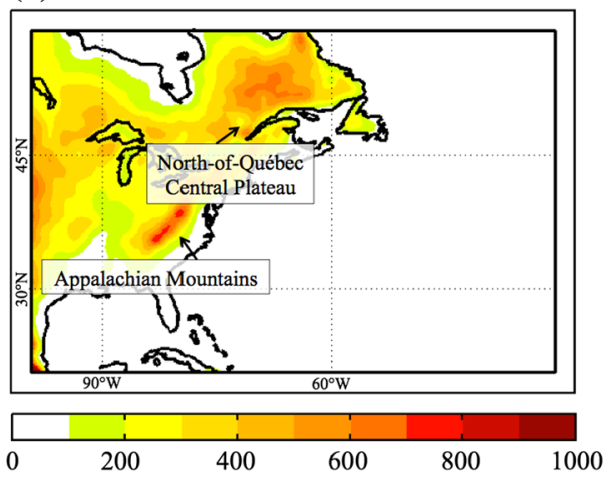

(c)

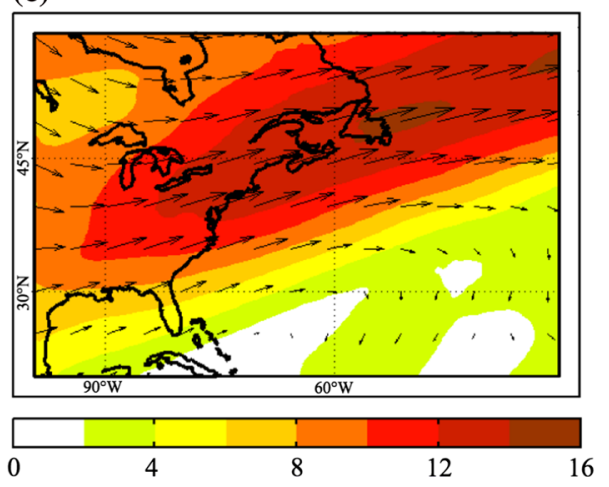

(b)

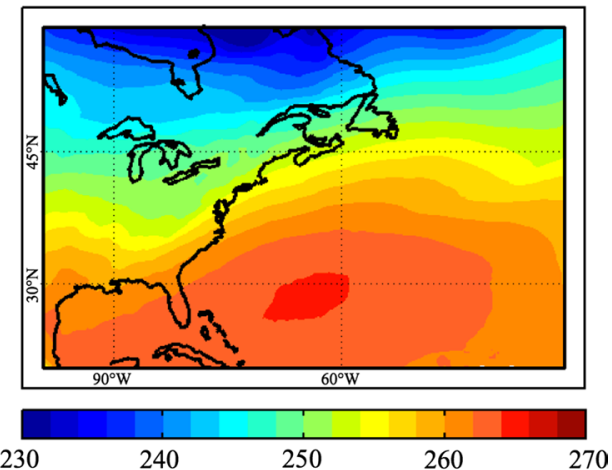

(d)

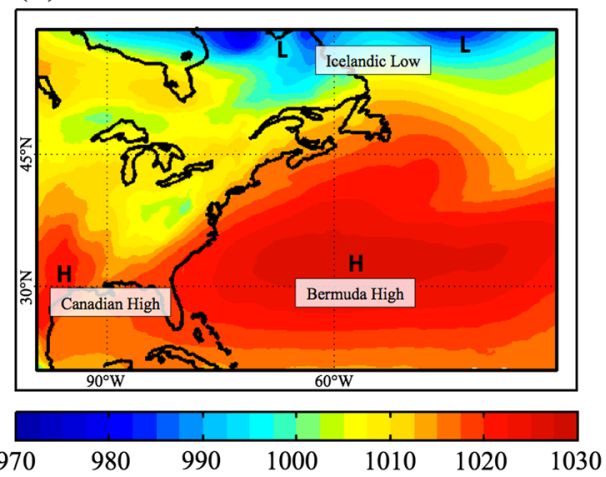

of available enthalpy. The terms $D$ represent dissipation of kinetic energy. The terms $F$ and $H$ are boundary fluxes and both would cancel out for an energy cycle computed over the whole globe. The terms $j$ that were present in the instantaneous energy cycle are here absent because they identically vanish upon time averaging.

\section{Experimental design}

The calculation of all the terms present in the energy equations requires several quantities that are either poorly or simply not observed, and often not routinely archived in reanalyses. The use of variables simulated by a high-resolution nested model driven by reanalyses circumvents this problem and also allows using higher temporal frequency than reanalyses.

\subsection{Simulation condition and domain}

A 1-year long simulation was made with the CRCM5 (Martynov et al. 2013) driven by ERA-interim available on a $1.5^{\circ}$ grid (Dee et al. 2011), starting on October 14 , 2004 at 0000 UTC. The month of December was chosen for the energy cycle budget calculations because of its important number of synoptic events typical of early winter conditions. The CRCM5 simulation was carried out on a non-rotated latitude and longitude grid with a grid mesh of $0.3^{\circ}, 56$ terrain-following hybrid levels in the vertical, and a time step of $12 \mathrm{~min}$. The CRCM5's code offers the option of large-scale spectral nudging, but this option was not turned on; in fact the simulation analysed here is one of those performed by Nikiema and Laprise (2016) to study the budget cycle associated with inter-member variability. The free domain, excluding the 10 grid-point-wide lateral Davies' sponge zone and the 10 grid-point-wide semiLagrangian halo, contains $230 \times 130$ grid points. It covers the east of the North American continent and a part of the western North Atlantic Ocean. It goes from $20.5^{\circ}$ to $59.2^{\circ}$ for the latitude and from $260.5^{\circ}$ to $329.2^{\circ}$ for the longitude. There are two main mountainous regions: the Appalachian Mountains and the North-of-Québec Central Plateau (Fig. 3a). The sea-surface temperature and sea-ice cover are prescribed from ERA-interim reanalyses. The simulated data is archived at a 3-hourly interval and the diagnostics are performed on variables interpolated on 18 pressure levels.

\subsection{Climate of December 2004}

Figure $3 b$ displays the vertically averaged time-mean temperature as simulated for the month of December 2004. There is a strong North-South temperature gradient across the domain and along the East Coast. The vertically 
Fig. 4 The period of occurrence of the storm studied is indicated by the black rectangle, corresponding to December 26-29, 2004. $p_{\text {smin }}$ shows the time evolution of the minimum value of surface pressure $(\mathrm{hPa})$ in the domain. $a_{T V \max }$ and $k_{T V \max }$ show the time evolution of the maximum value of available enthalpy and kinetic energy $\left(10^{5} \mathrm{~J} \mathrm{~m}^{-2}\right)$ in the domain

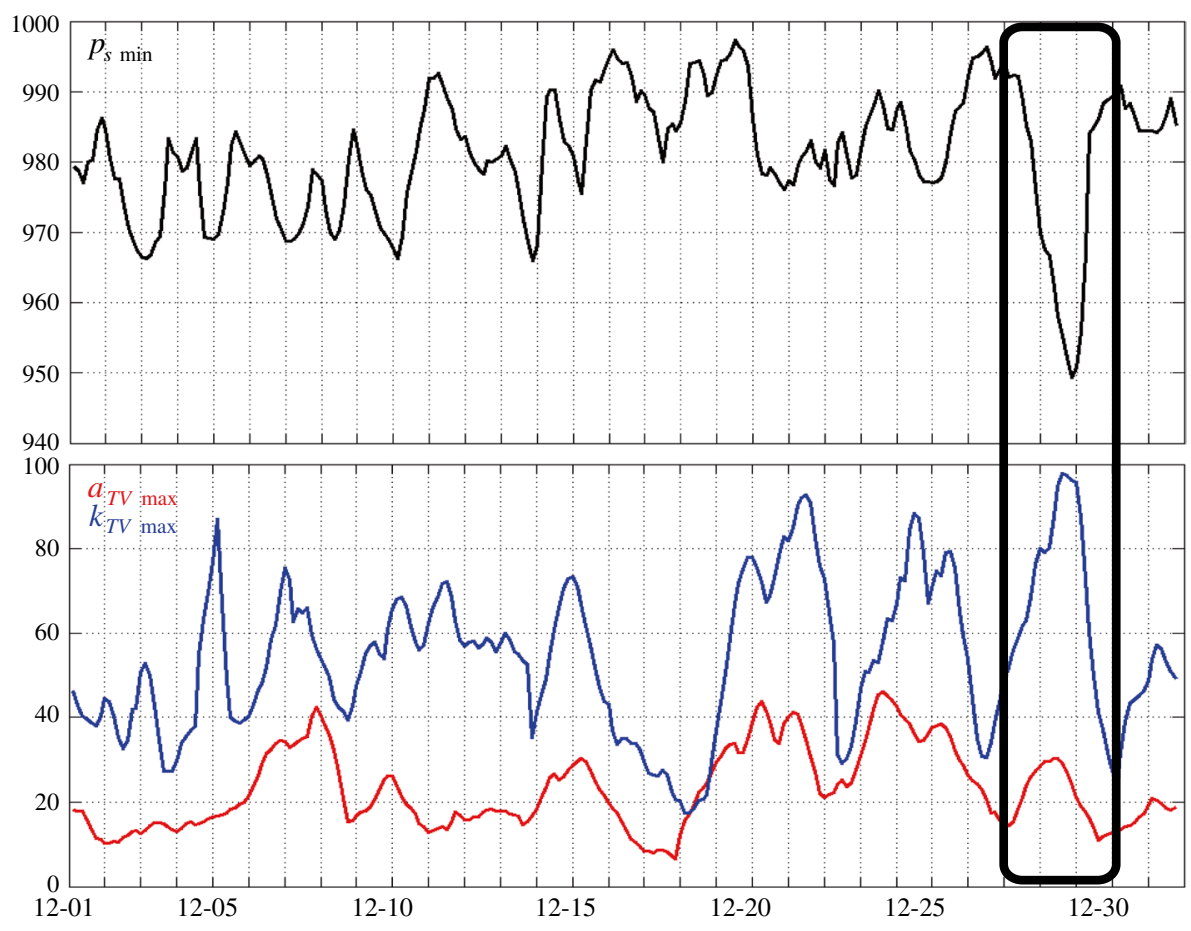

averaged time-mean wind pattern shown in Fig. $3 \mathrm{c}$ is well explained by the thermal wind relation, with the strongest wind being located over the largest horizontal temperature gradient. Climatological semi-permanent high- and lowpressure systems affect the weather in December: the Icelandic Low to the extreme northeast, the Canadian High in the west of the domain, and the Bermuda High in the east of the domain (Fig. 3d).

\section{Results and analysis of the storm energy cycle}

\subsection{Computation of the energy cycle contributions}

The time tendency $L_{E}=\partial E / \partial t$ of reservoirs $E \in\left\{a_{T V}, k_{T V}\right\}$ is computed as a centred time difference over a 6-hourly interval, using archived values of $E$ at 3-hourly intervals. The long-time average of $L_{E}$ is expected to be very small. $L_{E}$ is then compared to $R_{E}$, the sum of all sources and sinks of energy contributing to the tendency of $E$. In computing $R_{E}$, spatial derivatives are approximated as two-sided finite differences, and vertical integrals are computed with the trapezoidal rule after eliminating contributions below the surface. Formally $R_{E}$ should be identical to $L_{E}$ at all times. In practice however the equality is not exactly satisfied due to the numerous discretization approximations and interpolations. The terms $L_{E}$ and $R_{E}$ were computed for Eqs. (1, 2) for the month of December 2004 and were found have a correlation factor of $r \simeq 0.97$ (not shown), which implies that the diagnostically computed energy fluxes capture rather well the temporal variation of $E$ in the simulation. These results allow us to confirm the validity of the set of energy equations developed in Sect. 2.1 and to consider the instantaneous energy equations as being nearly satisfied.

\subsection{Choice of specific storm}

The storm chosen for the study occurred from December 26 to 29, 2004 in the analysis, and the corresponding CRCM5-simulated fields are used for the quantitative energy diagnostics. This specific storm was chosen because of its strong intensity (Fig. 4) and the fact that it developed and matured within the limited-area domain, making it an ideal case study of regional atmospheric energetics.

\subsection{Description of the storm}

Three moments were chosen at 24-hour intervals $\left(\mathrm{t}_{1}=2004-12-26, \mathrm{t}_{2}=2004-12-27\right.$ and $\mathrm{t}_{3}=2004-12-$ 28 , all at 2100 UTC) to illustrate the evolution of different terms through time. The storm started developing in the Atlantic Ocean, off the coast of Florida. It intensified while making its way northeastward along the East Coast of the United States, reached Nova Scotia and passed over Newfoundland. It reached the Labrador Sea while exiting the domain through the northeast boundary (Fig. 5). For $t_{1}$, the storm was in the Atlantic, on the East Coast of the United States with a central pressure value of $1004 \mathrm{hPa}$. For $\mathrm{t}_{2}$, it was in between Nova Scotia and Newfoundland, with a central pressure value of $986 \mathrm{hPa}$. Finally, for $\mathrm{t}_{3}$, it was 

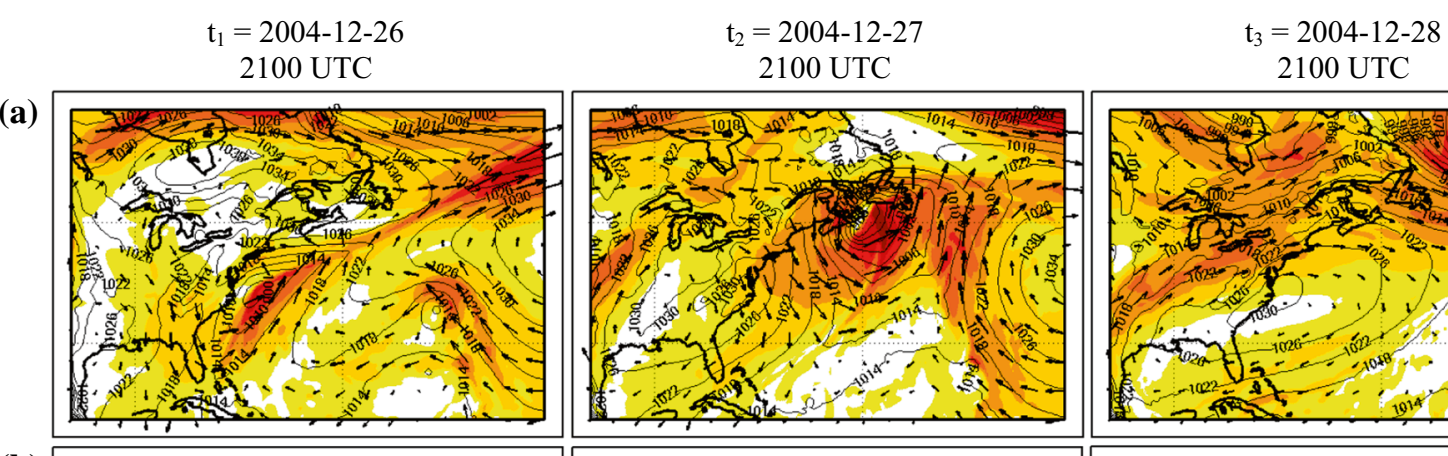

(b)
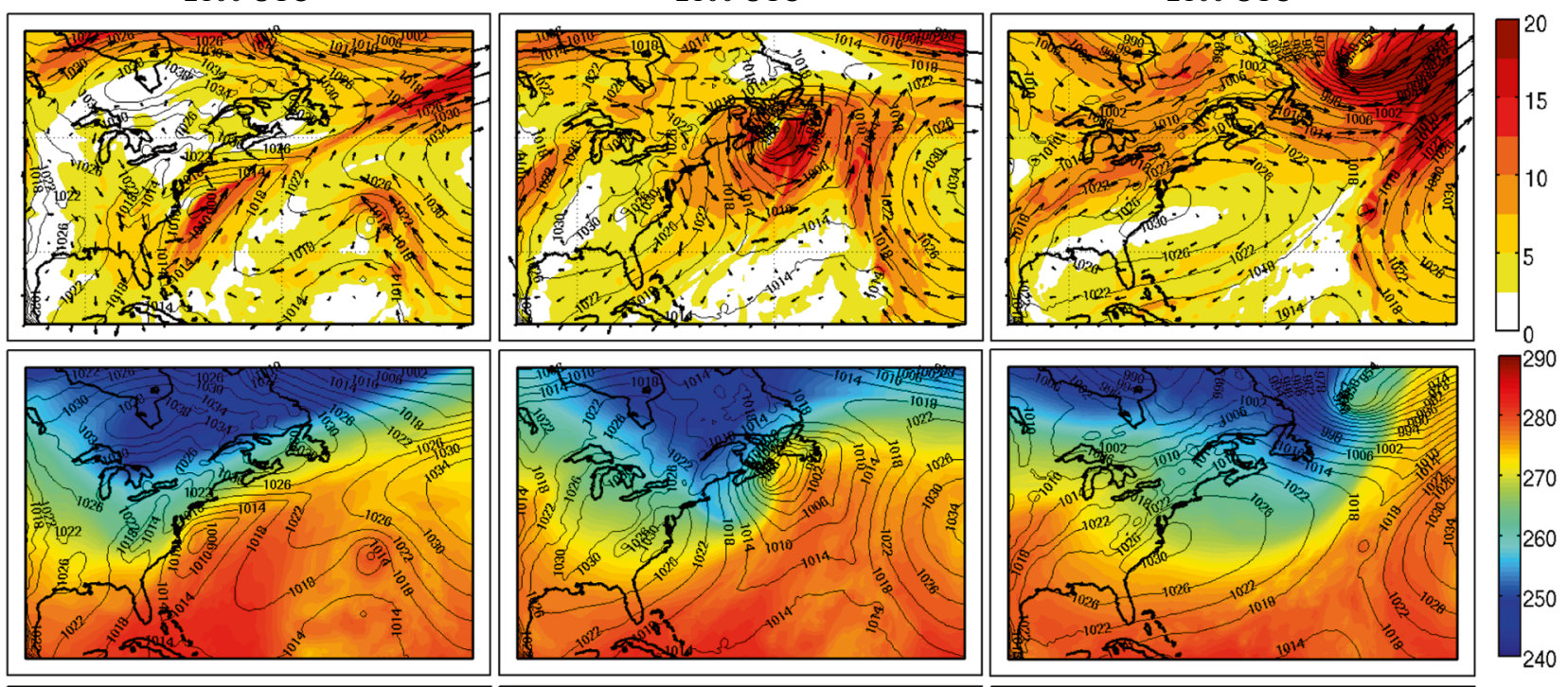

(c)
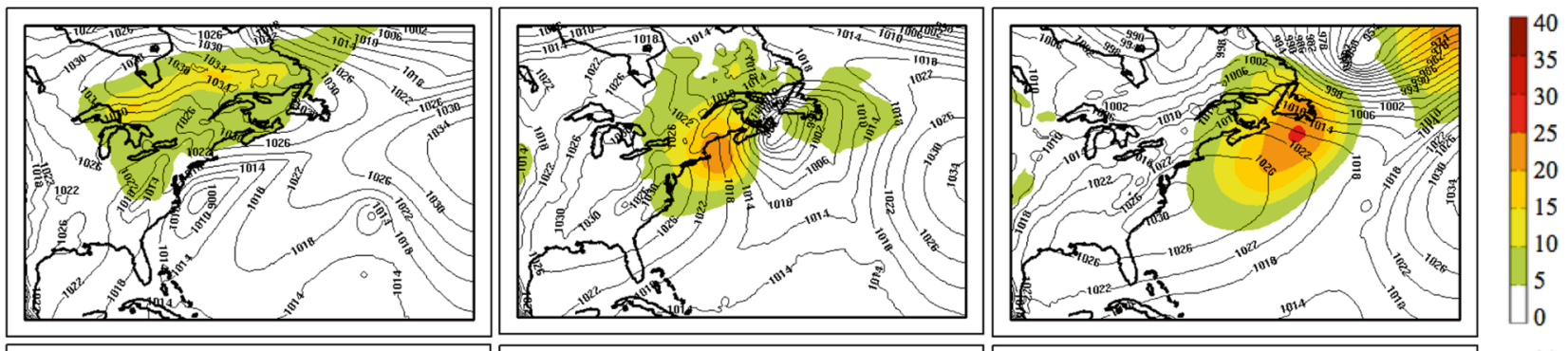

(d)
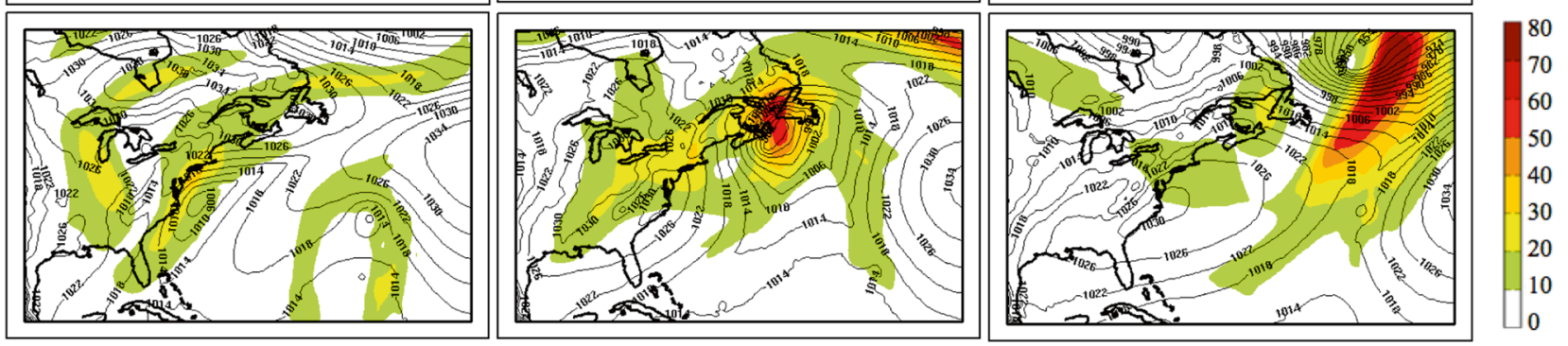

Fig. 5 Maps of instantaneous fields on three consecutive days of a 850-hPa horizontal wind $\vec{V}_{850}\left(\mathrm{~m} \mathrm{~s}^{-1}\right)$, b 700-hPa air temperature $T_{700}(\mathrm{~K})$, and vertical integrals of $\mathbf{c}$ time variability available enthalpy $a_{T V}\left(10^{5} \mathrm{~J} \mathrm{~m}^{-2}\right)$ and d) time variability kinetic energy $k_{T V}\left(10^{5} \mathrm{~J} \mathrm{~m}^{-2}\right)$

exiting the domain with a central pressure value of $954 \mathrm{hPa}$. The surface isobars are shown on all maps as a reminder of the storm's position. In Fig. 5 and in the following discussion, if there is no mention of a specific pressure level, it means that the field is vertically integrated from the surface to $200 \mathrm{hPa}$; this choice of upper integration limit will be discussed in Sect. 5.5.

The storm is associated with an important cyclonic circulation (Fig. 5a), where the wind is blowing southward on the west side of the low-pressure centre, and northward on the east side. The effect of the storm is very apparent on the lower atmosphere's temperature (Fig. 5b): cold air is carried southward on the west side and warm air is carried northward on the east side. The warm and cold fronts are very well defined, corresponding to zones of important horizontal temperature gradient and vertical velocity.

At the peak growth of the system (2004-12-27), there are two maxima of available enthalpy $a_{T V}$ (Fig. 5c): one located on the west side of the storm due to a cold air anomaly resulting from the cold air advection southward, the other located on its east side due to a warm air anomaly resulting from the warm air advection northward. The maximum of kinetic energy $k_{T V}$ (Fig. 5d) is located near the centre of the surface depression. As the depression core 

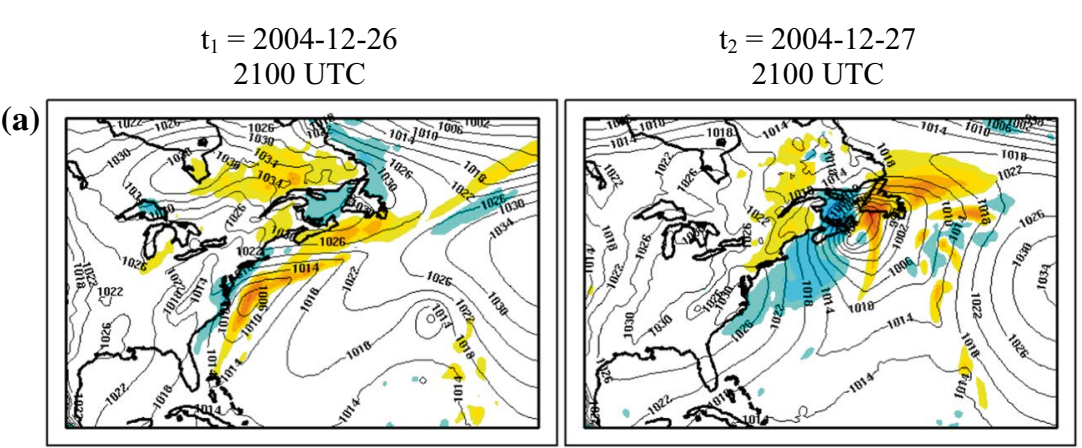

$\mathrm{t}_{3}=2004-12-28$

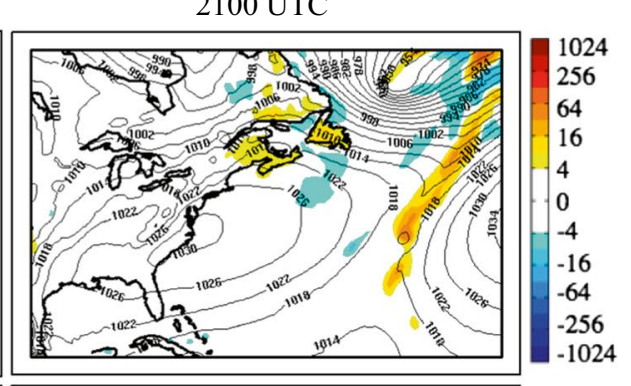

(b)
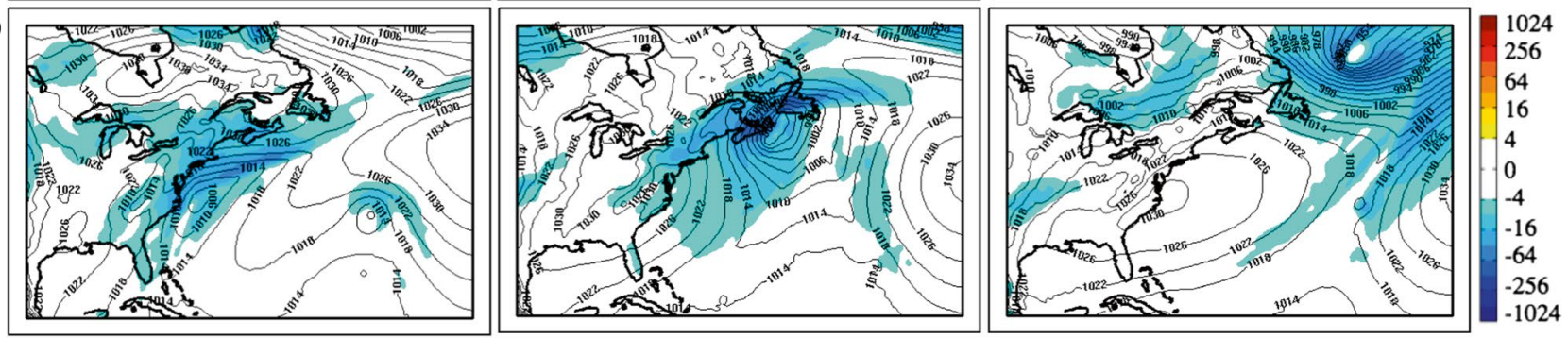

(c)
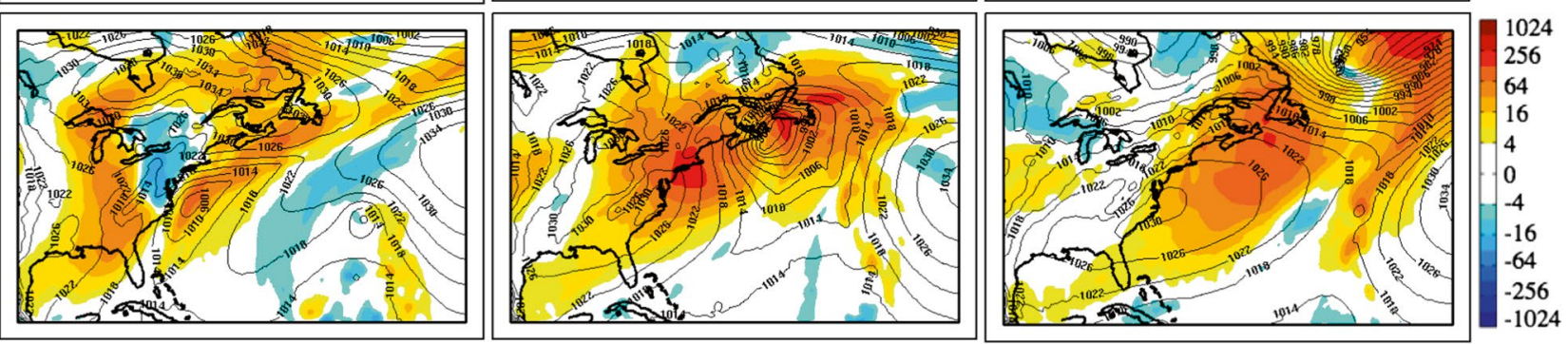

(d)
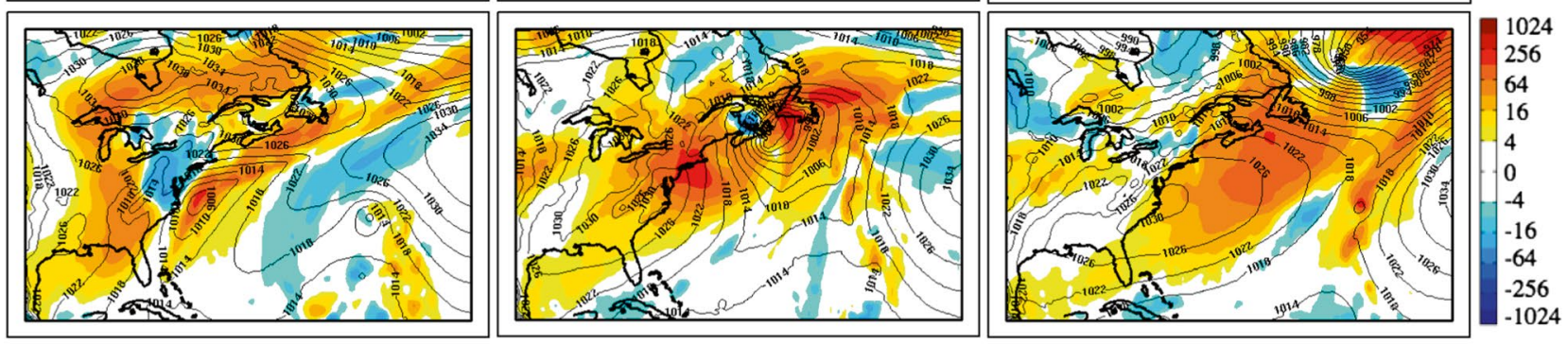

(e)
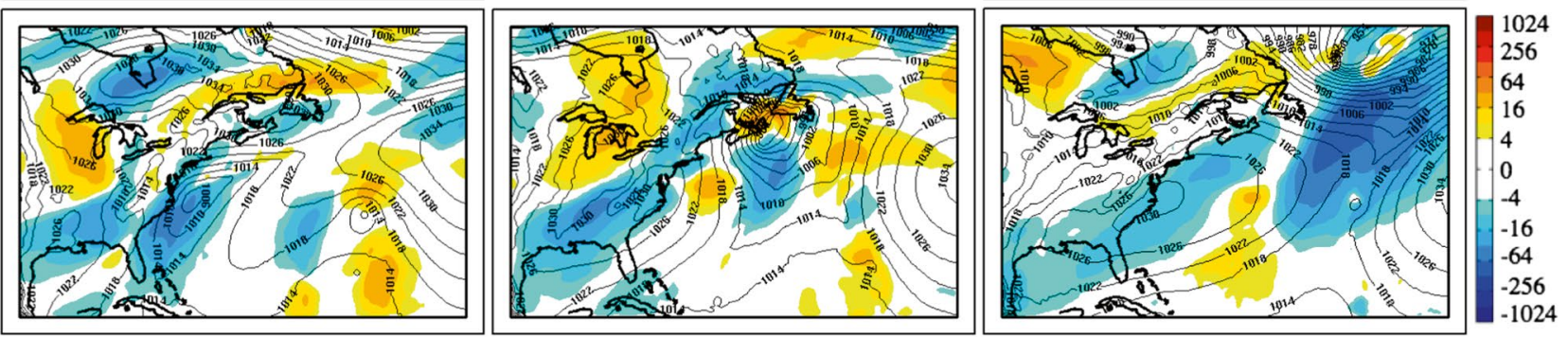

Fig. 6 Maps of instantaneous vertically integrated energy fluxes of a generation of time variability available enthalpy $g_{T V}$, b dissipation of time variability kinetic energy $-d_{T V}$, $\mathbf{c}$ conversion of time-mean into time variability available enthalpy $c_{a}$, $\mathbf{d}$ conversion of time variability

tilts westward with height, the surface depression is overlaid by the upper-level jet stream that undergoes large time variations as the system grows and moves. available enthalpy into time variability kinetic energy $c_{T V}$, and e conversion of time-mean into time variability kinetic energy $c_{k}$. Values are in $\mathrm{W} \mathrm{m}^{-2}$

Figures 6 and 7 show the various contributions to the time evolution of the energy reservoirs $a_{T V}$ and $k_{T V}$. The generation term $g_{T V}$ (Fig. 6a) results from the covariance 

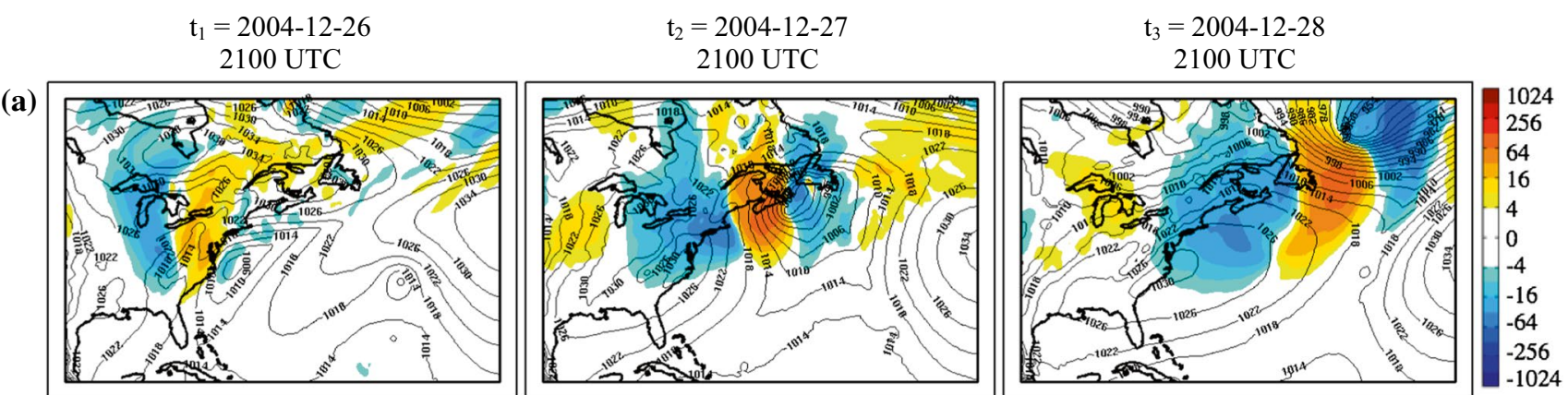

(b)
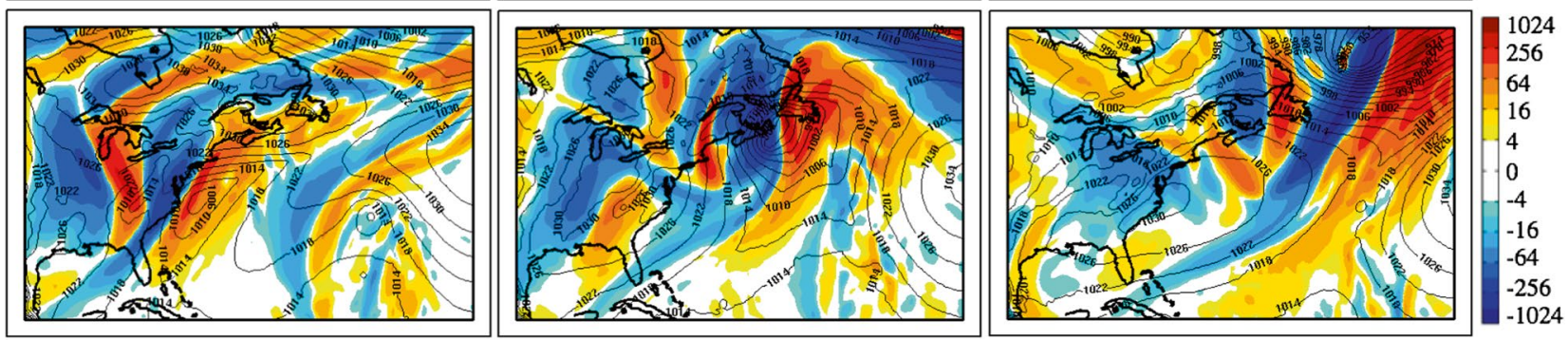

(c)
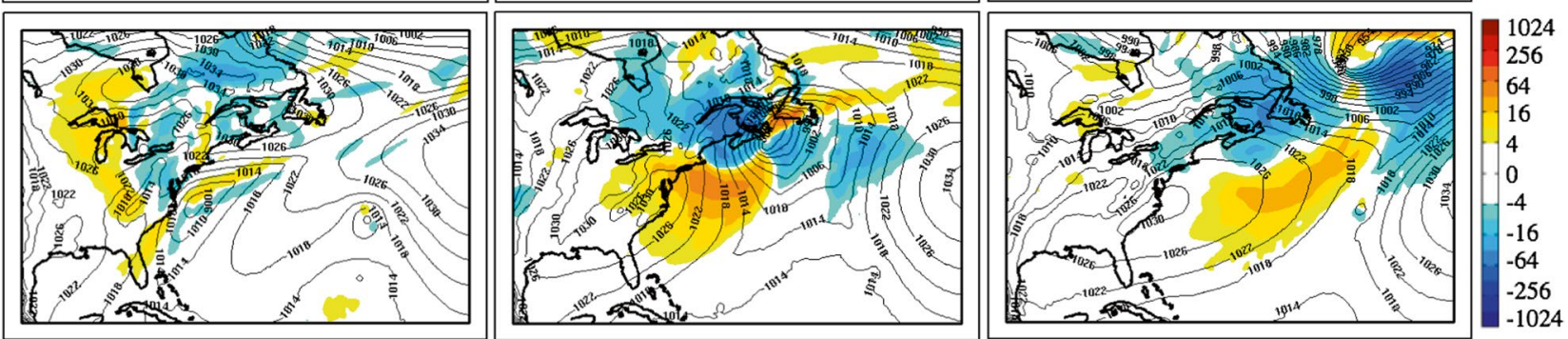

(d)
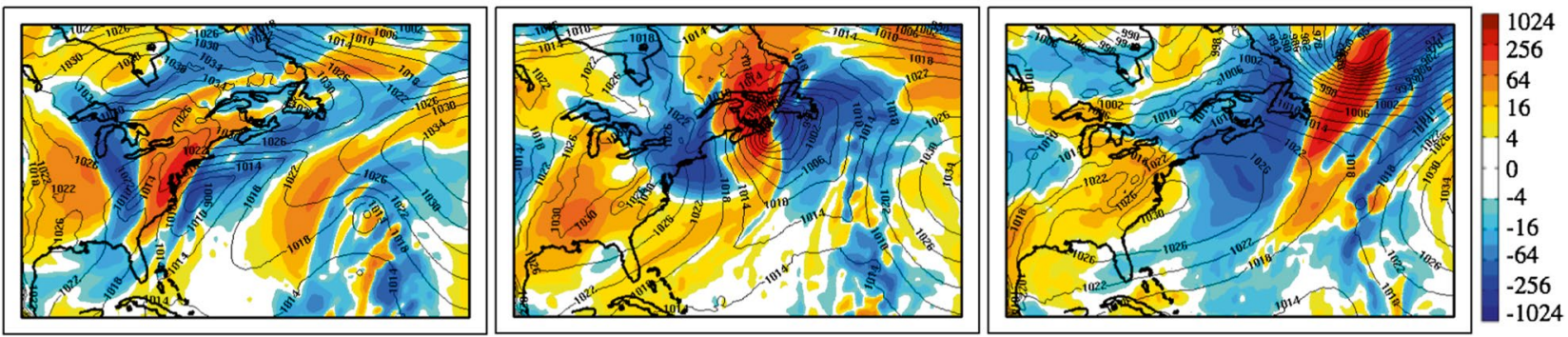

Fig. 7 Maps of instantaneous vertically integrated fluxes of transport of energy across lateral boundaries for a time variability available enthalpy $-f_{a_{T V}}$, b time variability kinetic energy $-f_{k_{T V}}$, c time vari-

of temperature and diabatic sources perturbations; the maximum of $g_{T V}$ follows the front lines where maximum convection and/or condensation occur. The dissipation term $d_{T V}$ (Fig. 6b) is maximum where the low-level wind and hence surface stress is maximum, which is around the storm where the isobars are the tightest. The term $\mathrm{c}_{\mathrm{a}}$ (Fig. 6c) converts time-mean available enthalpy $A_{T M}$ into time variability available enthalpy $a_{T V}$ by destroying the latitudinal temperature gradient and amplifying temperature anomalies through southward and downward transport of cold air on the west side of the storm and northward and upward transport of warm air on its east side, resulting in two dipoles of positive $c_{\mathrm{a}}$ in the domain. The term $c_{T V}$ ability available enthalpy $-h_{a_{T V}}$, and $\mathbf{d}$ time variability kinetic energy $-h_{k_{T V}}$. Values are in $\mathrm{W} \mathrm{m}^{-2}$

(Fig. 6d) is a baroclinic conversion of available enthalpy $a_{T V}$ into kinetic energy $k_{T V}$ as a result of warm anomalies rising and cold anomalies sinking. It is noteworthy that there is a great similitude between the pattern and intensity of the conversion terms $\mathrm{c}_{\mathrm{a}}$ and $c_{T V}$, which implies that most of the energy brought by $\mathrm{c}_{\mathrm{a}}$ to $a_{T V}$ is readily converted into $k_{T V}$ by $c_{T V}$. The term $c_{k}$ (Fig. 6e) corresponds to a barotropic conversion. There are several positive and negative patches in $c_{k}$, with negative areas dominating, indicating that the wind perturbations associated with the storm contribute to reinforcing the time-mean wind.

Figure 7 shows the contribution of terms that would vanish upon global integration, but that must be included in a 
regional energy budget. The transport terms $f_{a_{T V}}$ (Fig. 7a) and $f_{k_{T V}}$ (Fig. 7b) represent the fluxes of energy for $a_{T V}$ and $k_{T V}$, respectively; positive (negative) values are associated with the convergence (divergence) of energy by the timemean wind. By comparing these transport terms with the energy terms (see Fig. $5 \mathrm{c}$ for $a_{T V}$ and Fig. $5 \mathrm{~d}$ for $k_{T V}$ ), we see that a divergence-convergence dipole is centred on the maximum values of energy for $a_{T V}$ and $k_{T V}$; this indicates that the energy is being transported from zones of divergence toward zones of convergence, and this transport follows the general direction of the time-mean wind. The terms $h_{a_{T V}}$ (Fig. 7c) and $h_{k_{T V}}$ (Fig. 7d) are boundary fluxes for $a_{T V}$ and $k_{T V}$, respectively, and they both almost vanish when averaged over the domain. The terms $j_{a 1}, j_{a 2}$ and $j_{k 1}$ are negligible and hence are not shown.

\subsection{Summary of the energy path}

In Fig. 1 are presented the domain-averaged and vertically integrated values of the energy reservoirs and energy fluxes, which have been averaged over the 2 days of the storm. We see that most of the energy is converted from the timemean available enthalpy $A_{T M}$ into its time variability state $a_{T V}$ by the conversion term $\mathrm{c}_{\mathrm{a}}$, while the time variability available enthalpy $a_{T V}$ is simultaneously converted into the time variability kinetic energy $k_{T V}$ by the baroclinic conversion $c_{T V}$. Some of this time variability kinetic energy $k_{T V}$ is then converted to feed the time-mean kinetic energy $K_{T M}$ by the barotropic conversion $c_{k}$, some is dissipated by $d_{T V}$ and some is transported out of the domain by the transport term $f_{k_{T V}}$. It is interesting to note that the ratio $k_{T V} / c_{T V}=1.1$ day; this means that if the baroclinic conversion $c_{T V}$ from time variability available enthalpy $a_{T V}$ were the only term contributing to the growth of time variability kinetic energy $k_{T V}$, it would grow with an e-folding time of 1.1 day, which appears a typical baroclinic growth rate.

\section{Results and analysis of the energy cycle for the month of December 2004}

We will now analyse the atmospheric energetics calculated over the entire month of December 2004, as an illustration of the climatological winter energy budget of an extra-tropical North American region.

\subsection{Computation of the energy cycle contributions}

The energies in the six reservoirs $E \in\left\{B, A_{T M S}, A_{T M B}, A_{T V}, K_{T M}, K_{T V}\right\}$ were computed as well as the various energy fluxes. The terms $L_{E}$ were computed and, as expected, their monthly means were found to nearly vanish (not shown). The monthly means of $R_{E}$ were also found to be very small and negligible in comparison with the diagnostically computed energy fluxes acting upon $E$ in the simulation. These results allow us to confirm the accuracy of the calculations performed using the set of energy equations developed in Sect. 2.2.

\subsection{Maps of the energy reservoirs}

Figure 8 shows the time-averaged vertically integrated maps of the six energy reservoirs developed in Sect. 2, respectively a) $B$, b) $A_{T M S}$, c) $A_{T M B}$, d) $K_{T M}$, e) $A_{T V}$ and f) $K_{T V}$, (note the different scales). As expected, the pressure-dependent available enthalpy $B$ (Fig. 8a) exhibits smaller values where the surface pressure is lower on average, such as over high topography and over the Icelandic Low (see Fig. 3a, d). The time-mean stratification available enthalpy $A_{T M S}$ (Fig. $8 \mathrm{~b}$ ) reflects the mean temperature state of the atmosphere (see Fig. $3 b$ ): cold in high latitudes (low available enthalpy) and warm in the tropics (high available enthalpy). The time-mean baroclinic available enthalpy $A_{T M B}$ (Fig. 8c) reflects the temperature variance with respect to the isobaric temperature average: cold high latitudes and warm low latitudes resulting in high values of $A_{T M B}$, while the middle of the domain is close to the isobaric mean temperature value, resulting in small value of $A_{T M B}$. The time-mean kinetic energy $K_{T M}$ (Fig. 8d) reflects the time-mean flow speed (see Fig. 3c), with the maximum kinetic energy being located where the strongest wind is. The maximum temperature and wind variations occur along the storm track, contributing to the time variability available enthalpy $A_{T V}$ (Fig. 8e) and the time variability kinetic energy $K_{T V}$ (Fig. 8f), respectively.

\subsection{Maps of the energy fluxes}

Figure $9 \mathrm{a}, \mathrm{b}$ show the time-averaged vertically integrated maps of the energy fluxes acting on the energy reservoirs (note the non-linear colour scale). The energy cycle has been split into two figures for better representation of the energy fluxes maps, but both figures show sections of the same energy cycle. The main energy conversion terms presented in Fig. 9 are analysed in Sect. 5.4.

The terms $G_{T M S}, G_{T M B}$ and $G_{T V}$ are generation terms acting on available enthalpy reservoirs $A_{T M S}, A_{T M B}$ and $A_{T V}$, respectively. The generation term $G_{T M S}$ is due to the covariance of diabatic sources and temperature; it is positive over the storm track and the ocean, which implies diabatic heating (cooling) in regions that are warmer (colder) than the reference temperature. The terms $G_{T M B}$ and $G_{T V}$ are due to the covariance of diabatic sources and temperature deviations, which are small for mid-latitudes in winter. 
(a)
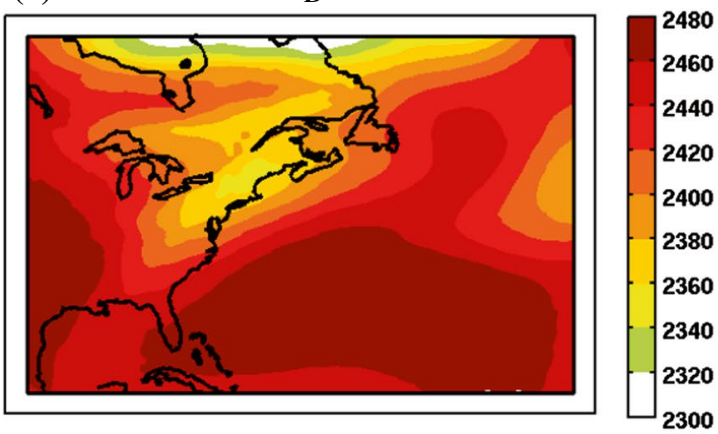

(c)
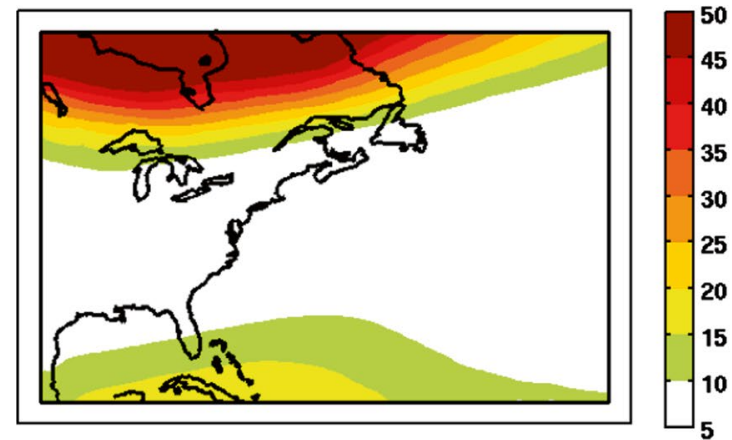

(e)
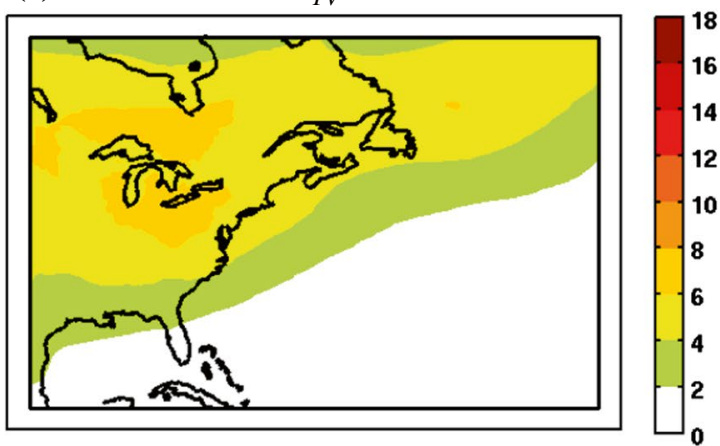

(b)

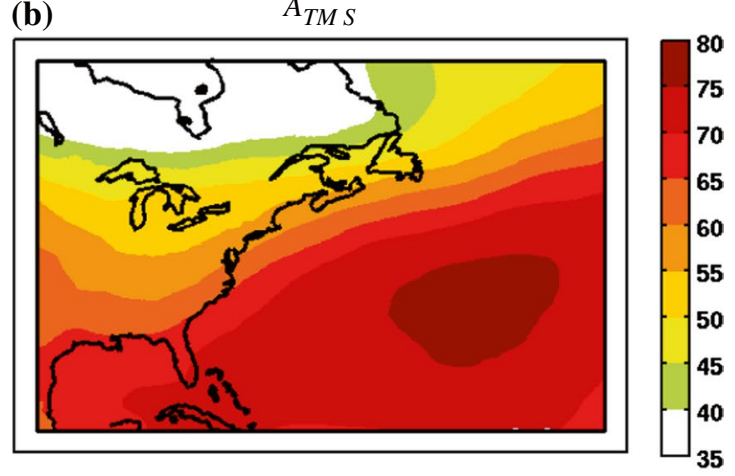

(d)
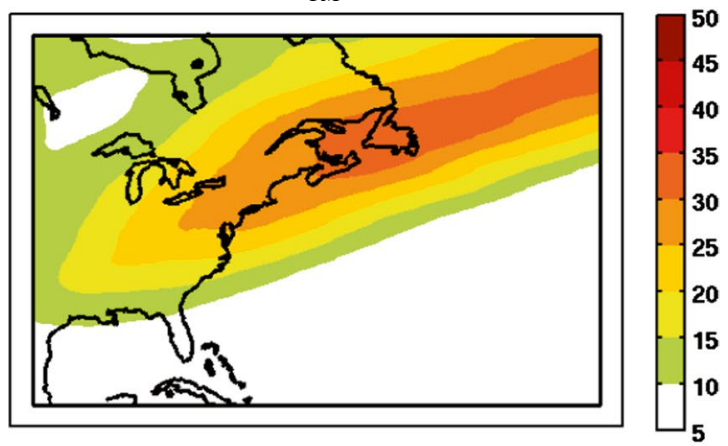

(f)
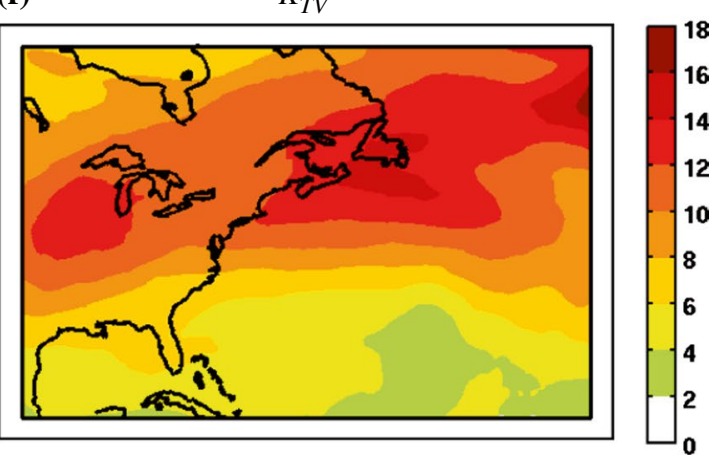

$A_{T M B}$, d time-mean kinetic energy $K_{T M}$, e time variability available enthalpy $A_{T V}$, and $\mathbf{f}$ time variability kinetic energy $K_{T V}$. a, b have different scales, $\mathbf{c}$, $\mathbf{d}$ have the same scale and $\mathbf{e}, \mathbf{f}$ have another scale. Values are in $10^{5} \mathrm{~J} \mathrm{~m}^{-2}$

around the perimeter, with the downward contribution dominating. Hence, $I_{A B}$ is negative and it converts a remarkably high amount of available enthalpy from $A_{T M}$ to $B$.

Fig. 9 Maps of vertically integrated time-averaged energy fluxes $\left(\mathrm{W} \mathrm{m}^{-2}\right)$ between the various energy reservoirs. a Shows the fluxes for the pressure-dependant part of available enthalpy reservoir $B$ and the stratification component of available enthalpy reservoir $A_{T M S}$. b Shows the fluxes for time-mean and time variability available enthalpy reservoirs $A_{T M B}$ and $A_{T V}$, and time-mean and time variability kinetic energy reservoirs $K_{T M}$ and $K_{T V}$. Note that the scale is nonlinear. The isobaric-mean value for each flux can be found in Fig. 2 
(a)
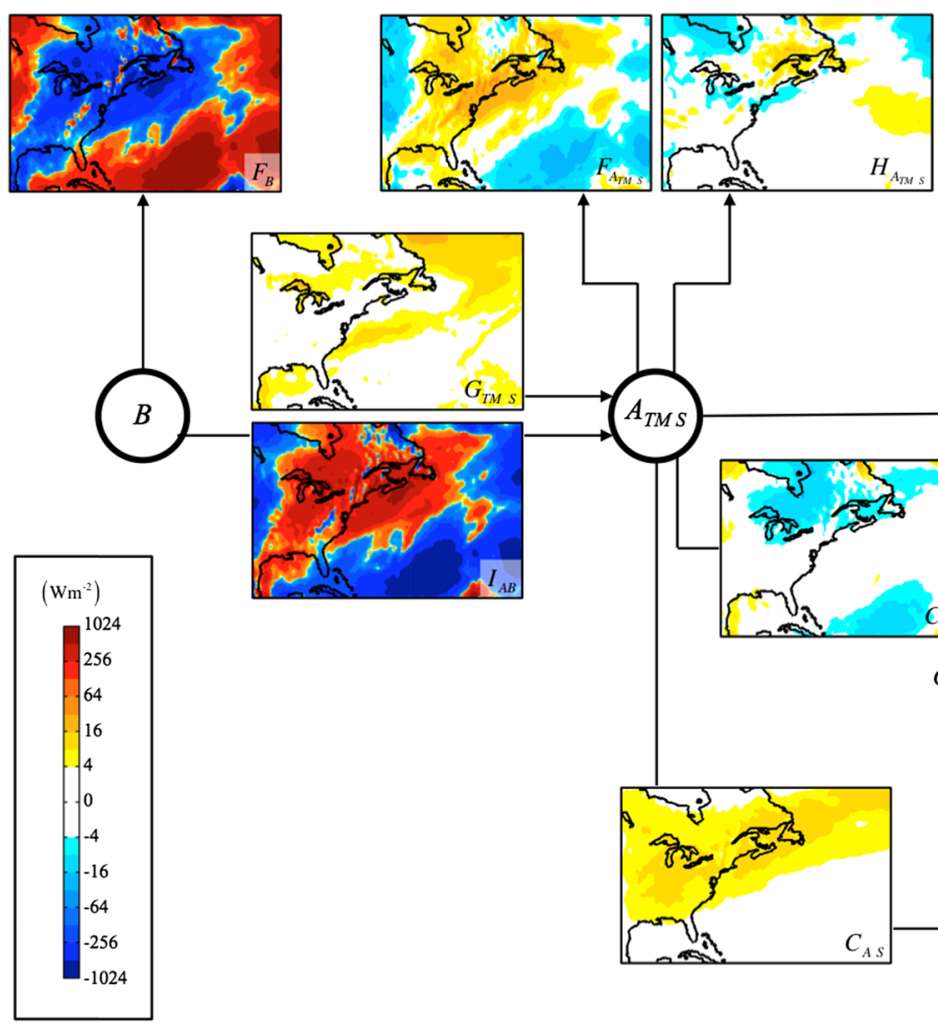

(b)

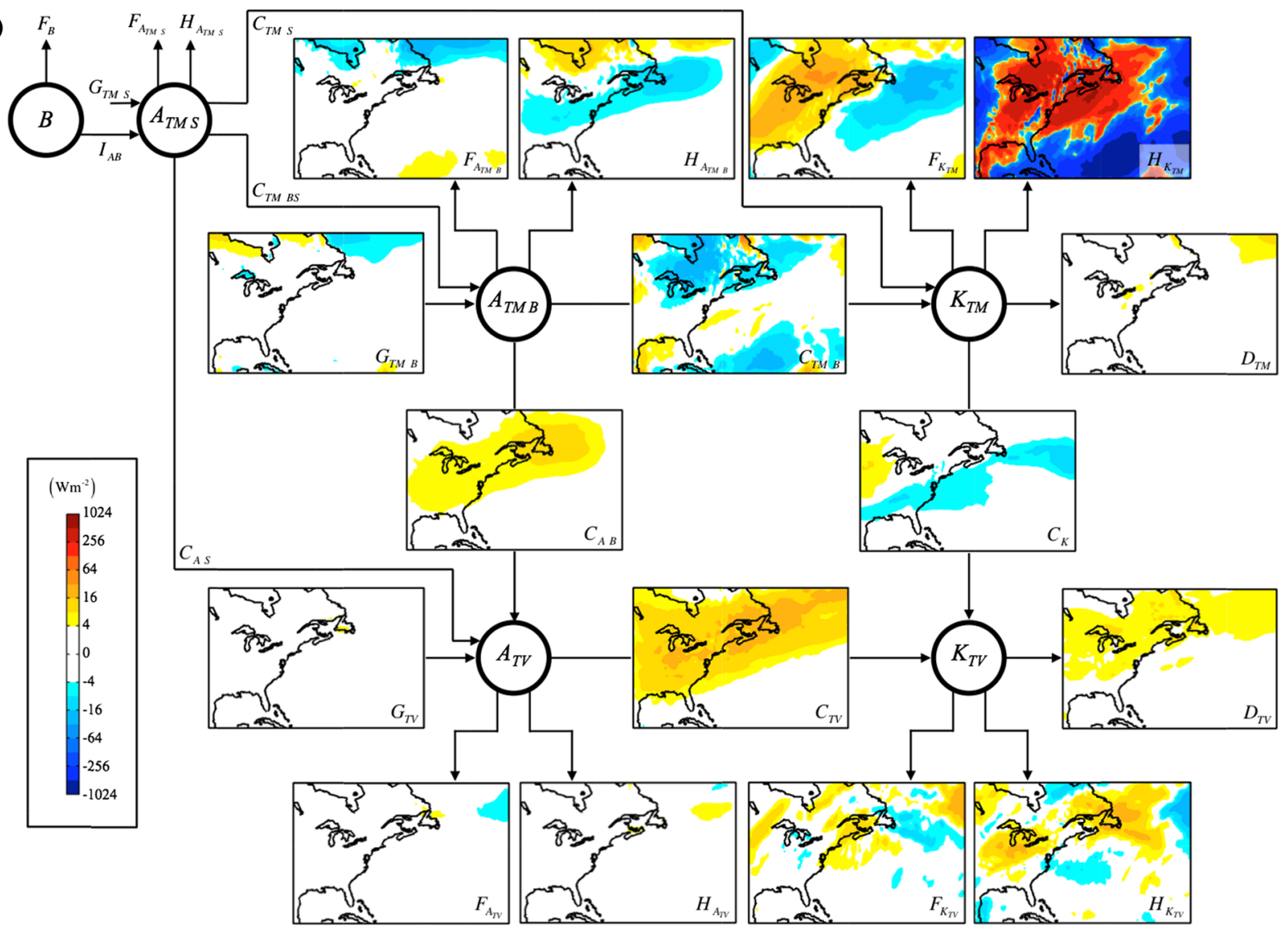


Fig. 10 Maps of time-averaged vertically integrated energy conversions of a time-mean into time variability available enthalpy $C_{A}$, b time-mean into time variability kinetic energy $C_{K}$, c time-mean available enthalpy into time-mean kinetic energy $C_{T M}$, and $\mathbf{d}$ time variability available enthalpy into time variability kinetic energy $C_{T V}$. Values are in $\mathrm{W} \mathrm{m}^{-2}$ (a)

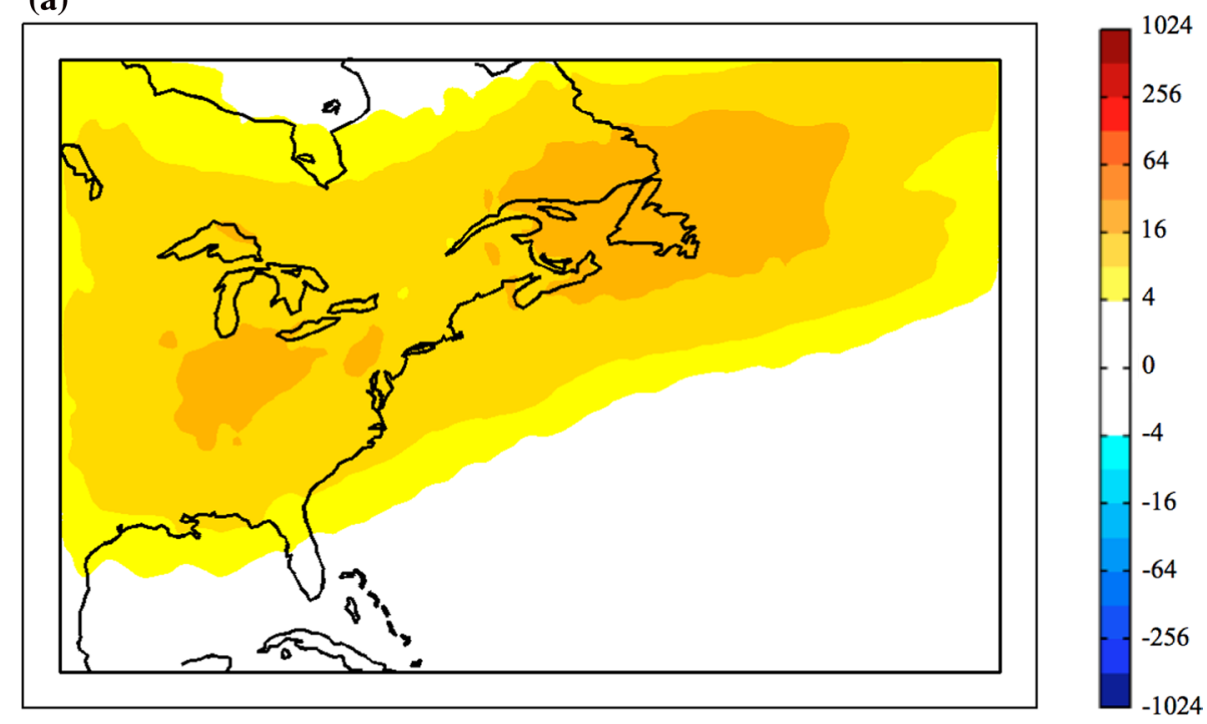

(b)

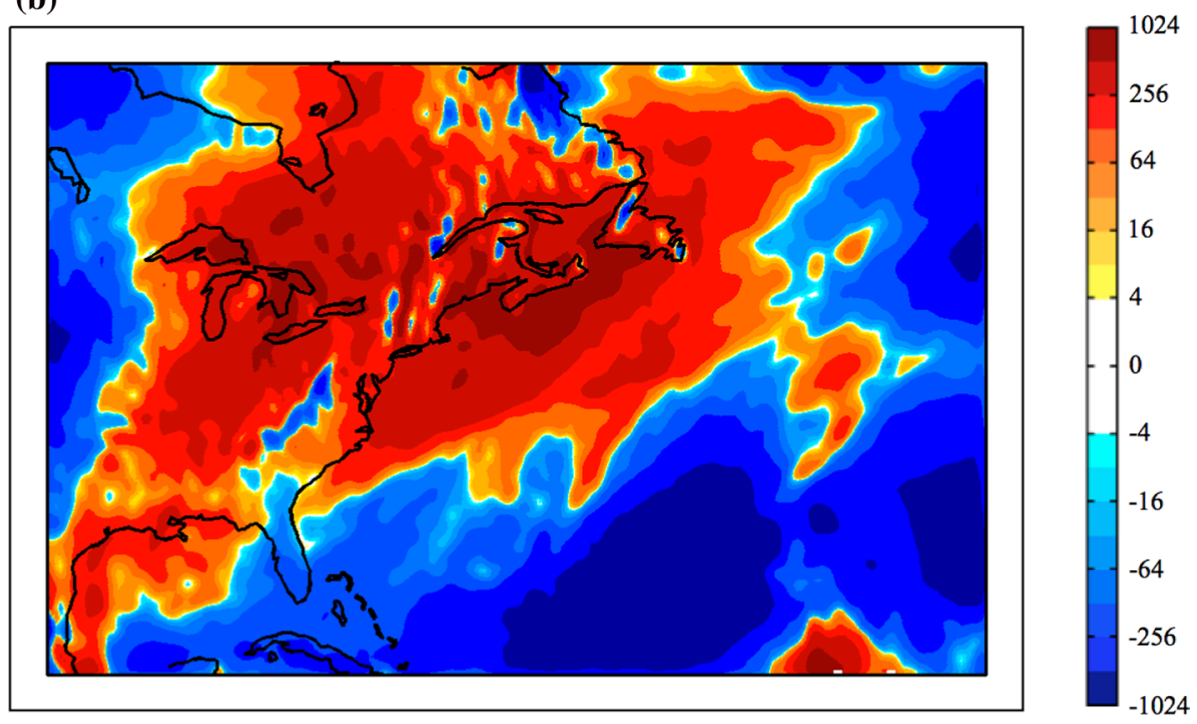

The term $C_{T M B S}$ represents a conversion of time-mean available enthalpy between its stratification component $A_{T M S}$ and its baroclinic component $A_{T M B}$. We note from the map that the field is negative, indicating that the conversion is from $A_{T M B}$ to $A_{T M S}$ over the period and domain of interest.

The six terms $F$ are boundary fluxes representing the transport of energy in and out of the limited-area domain by the time-mean flow. These terms would vanish upon integrating over the entire atmosphere. Over the period and domain of interest, they act overall as source for the reservoir $A_{T M B}$ and sink for reservoirs $B, A_{T M S}, K_{T M}, A_{T V}$ and $K_{T V}$ (see Fig. 2). The five terms $H$ are also boundary fluxes that would vanish upon integrating over the entire atmosphere. Over the period and domain of interest, they act overall as a source of energy for reservoirs $A_{T M B}$ and $K_{T M}$ and as a sink of energy for reservoirs $A_{T M S}, A_{T V}$ and $K_{T V}$. The fluxes $F_{A_{T V}}$ and $H_{A_{T V}}$ are however very small. The specific values of the boundary fluxes $F$ and $H$ are consequences of the flow over the chosen period of time and domain, and for different periods or different regions, these results could be very different. They can act either as sink or source since energy can be lost or gained by exchanges between the regional and external domains.

The four terms $F_{B}, I_{A B}, C_{T M} S$ and $H_{K_{T M}}$ look very similar and they transport a remarkably high amount of energy due to the important values of $B$ and $\langle\Phi\rangle$, respectively.

\subsection{Maps of the energy conversions}

Figures $9 \mathrm{~b}$ and 10 present the four main energy conversion terms, namely $C_{A}, C_{T V}, C_{K}$ and $C_{T M}$. The term $C_{A}$ (Fig. 10a) 
is the sum of $C_{A S}$ and $C_{A B}$ (individually shown in Fig. 9b), converting available enthalpy from the time-mean state $A_{T M S}$ and $A_{T M B}$ into the time variability state $A_{T V}$, respectively. $C_{A}$ represents the effect of the covariance of wind and temperature perturbations interacting with the timemean temperature gradient. The temperature and wind patterns of December 2004 (see Fig. 3b, c) transport cold air south-eastward and warm air north-westward, hence down the time-mean temperature gradient, making the conversion term $C_{A}$ contribute positively. The energy conversion is largest where the temperature gradient is the strongest, along the storm track.

The term $C_{T V}$ (Fig. 9b) converts time variability available enthalpy $A_{T V}$ into time variability kinetic energy $K_{T V}$. It represents a baroclinic conversion due to the covariance of vertical velocity and temperature (specific volume) perturbations. This conversion is everywhere positive over the period and domain of interest, indicating that warm (cold) anomalies are associated with upward (downward) vertical velocity anomalies. Indeed, $C_{T V}$ is largest along the storm track where fronts are associated with large temperature and vertical velocity perturbations. If we compare the conversions terms $C_{T V}$ and $C_{A}$, we note a great similarity between the patterns and intensity of the conversion terms, indicating that most of the energy brought by $C_{A}$ to time variability available enthalpy $A_{T V}$ is readily converted by $C_{T V}$ into time variability kinetic energy $K_{T V}$.

The barotropic term $C_{K}$ (Fig. 9b) represents a conversion of kinetic energy between its time-mean state $K_{T M}$ and its time variability state $K_{T V}$. It represents the effect of the variance and covariance of horizontal wind perturbations interacting with the time-mean horizontal wind gradient. The field of $C_{K}$ is negative and located over the storm track, corresponding to a conversion from $K_{T V}$ into $K_{T M}$ and indicating that wind perturbations contribute to reinforce the time-mean wind.

The term $C_{T M}$ (Fig. 10b) is the sum of $C_{T M S}$ and $C_{T M B}$ (individually presented in Fig. 9b), converting time-mean available enthalpy $A_{T M S}$ and $A_{T M B}$ into time-mean kinetic energy $K_{T M}$, respectively. Compared to $C_{T M B}$, the conversion term $C_{T M S}$ exhibits a large magnitude, of the same order of magnitude as $I_{A B}$ (see Fig. 9a). On average, both terms are negative, making $C_{T M}$ also mainly negative, indicating that $K_{T M}$ converts energy into $A_{T M S}$ and $A_{T M B}$. The term $C_{T M}=-\langle\omega\rangle\langle\alpha\rangle$ exhibits mostly positive values in the middle part of the domain, indicating that time-averaged upward motion of warm air and downward motion of cold air. In the southern part of the domain, large negative values seem to be associated with downward motion of warm air around the Bermuda High (Fig. 3d). Overall, the timemean wind contributes to increase the time-mean temperature gradient over the period and domain of interest.

\subsection{Vertical profiles}

Figure 11 shows the vertical profiles of the time- and isobaric-averaged fluxes for the six energy reservoirs, respectively a) $B$, b) $A_{T M S}$, c) $A_{T M B}$, d) $K_{T M}$, e) $A_{T V}$ and f) $K_{T V}$. The vertical profiles are shown from 1000 to $50 \mathrm{hPa}$. As can be noted in most panels, the behaviour of fields above the $200 \mathrm{hPa}$ level differs markedly from their behaviour in the troposphere where most of the weather phenomena occur; this motivated our choice of restricting the vertical integration up to the $200 \mathrm{hPa}$ level so as to keep the analysis focused upon meteorological events.

The thick black lines represent $R_{E}$, the algebraic sum of all energy fluxes acting on $E$. For the three time-mean state reservoirs $B, A_{T M S}$ and $K_{T M}$, some terms exhibit remarkably higher values, namely $I_{A B}, C_{T M S}, F_{B}$ and $H_{K_{T M}}$, with an almost perfect balance between them (see also their maps in Fig. 9) and the other terms are negligible. The four terms represent the conversions of time-mean energy in between the three reservoirs by $I_{A B}$ and $C_{T M S}$, and the transport of that energy through the domain boundaries by $F_{B}$ and $H_{K_{T M}}$. For the time-mean baroclinic available enthalpy $A_{T M B}$, energy fluxes of lesser amplitude interact to balance each other. In the lower part of the troposphere, the conversion term $-C_{A B}$ is balanced by the boundary

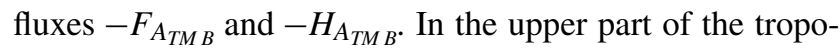
sphere, $C_{T M B S}$ is balanced by $-C_{T M B}$, which means that the energy converted from $K_{T M}$ to $A_{T M B}$ is then converted to $A_{T M S}$. For the time variability available enthalpy $A_{T V}$, the conversion term $-C_{T V}$ is almost balanced by conversion terms $C_{A}=C_{A S}+C_{A B}$ in the free atmosphere. As mentioned in Sect. 5.4, this result confirms that the energy converted from $A_{T M}$ to $A_{T V}$ by $C_{A}$ is readily converted by $C_{T V}$ into $K_{T V}$. Physically, this is equivalent to a baroclinic conversion from eddy $A P E$ to eddy $K E$ (e.g. Lorenz 1955 , 1967). The negative value of the baroclinic conversion term $C_{T V}=-\left\langle\omega^{\prime} \alpha^{\prime}\right\rangle$ reflects the fact that perturbations of vertical velocity and temperature are negatively correlated: upward (downward) vertical velocity perturbations are associated with warm (cold) perturbations. Both have maximum intensities at mid troposphere; indeed, the covariance of vertical velocity and temperature perturbations $C_{T V}$ is maximum where the vertical velocity $\omega$ is maximum, near $p \simeq 400 \mathrm{hPa}$. The vertical transport of temperature (which dominates over the horizontal transport in $C_{A S}+C_{A B}$ ) is also maximum at this level.

The negative value of the production term $G_{T V}$ in the boundary layer is due to surface sensible heat flux and vertical diffusion. The other terms are negligible. For the time variability kinetic energy $K_{T V}$, the baroclinic conversion $C_{T V}$ is balanced by the boundary flux term $-H_{K_{T V}}$ in the free atmosphere. In the boundary layer however the balance is entirely different, with the dissipation term $-D_{T V}$ 

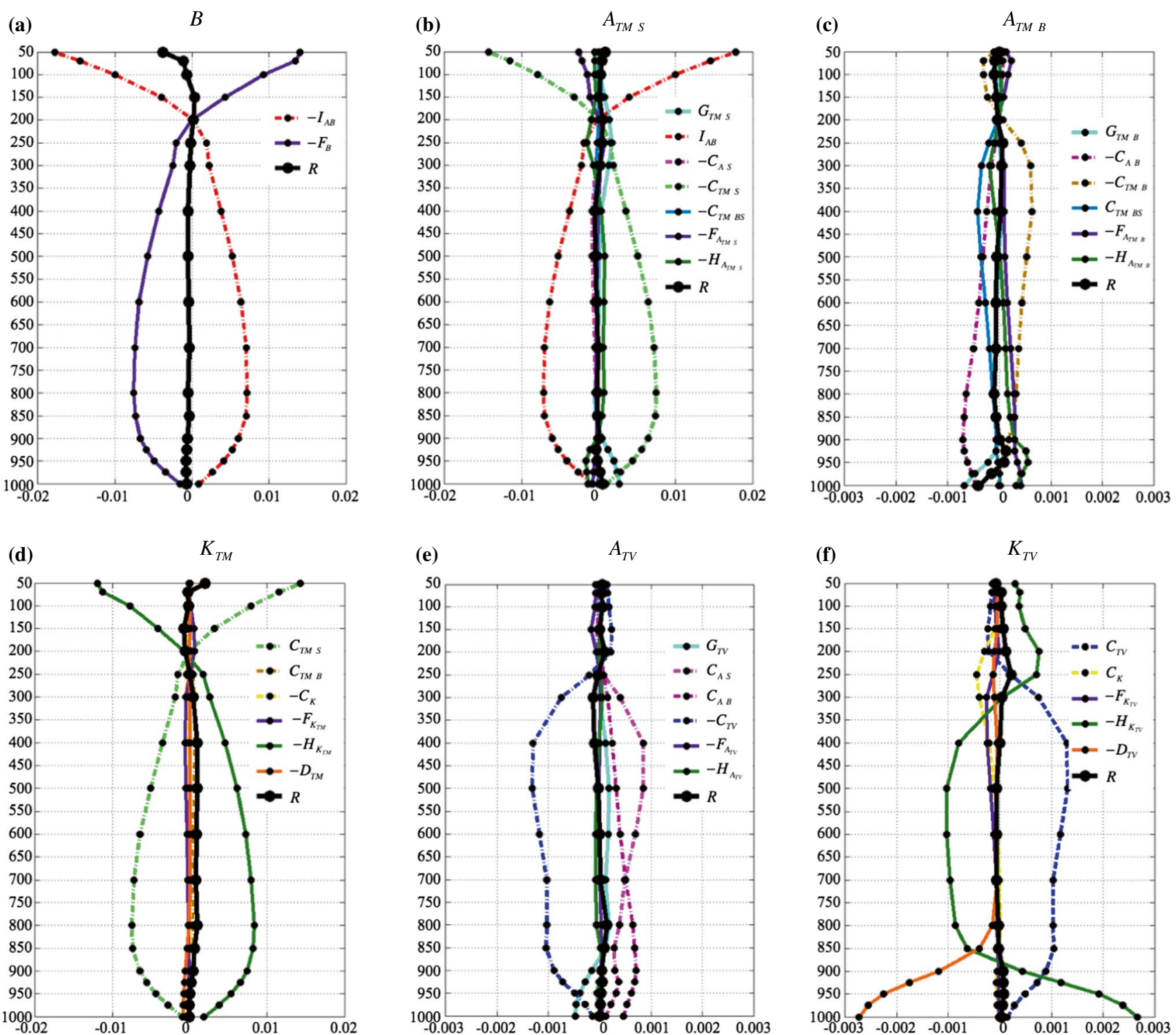

Fig. 11 Vertical profiles of time- and domain-averaged fluxes acting on energy reservoirs of a pressure-dependant part of available enthalpy $B$, b stratification component of available enthalpy for the time-mean state $A_{T M S}, \mathbf{c}$ baroclinic component of available enthalpy for the time-mean state $A_{T M B}$, d time-mean kinetic energy $K_{T M}$, e time variability available enthalpy $A_{T V}$, and $\mathbf{f}$ time variability kinetic energy $K_{T V}$. The abscissa is the energy flux in $\mathrm{W} \mathrm{kg}^{-1}$ and the ordinate is the pressure in $\mathrm{hPa}$. $\mathbf{a}, \mathbf{b}$, and $\mathbf{d}$ have the same scale and $\mathbf{c}, \mathbf{e}$ and $\mathbf{f}$ have another scale due to surface friction being balanced by the component of $-H_{K_{T V}}$ corresponding to the covariance of vertical velocity and geopotential height perturbations, $\frac{\partial}{\partial p}\left\langle\Phi^{\prime} \omega^{\prime}\right\rangle$. In low (high) pressure systems, surface friction induces convergence (divergence) of mass down (up) the gradient of geopotential, resulting in upward (downward) motion, a process known as Ekman pumping (e.g. Holton 2004, Sect. 5.4). Hence in the boundary layer, the energy lost by friction $\left(-D_{T V}\right)$ is balanced by the energy converted by Ekman pumping $\left(-H_{K_{T V}}\right)$. The other terms in the budget of the time variability kinetic energy $K_{T V}$ are negligible.

\subsection{Summary of the energy path}

As discussed in Sect. 2, this regional atmospheric budget admits six energy reservoirs unlike the four normally discussed for global energetics. The additional two reservoirs $B$ and $A_{T M S}$ arise because of the non-conservation of the mass over a limited domain $(B)$, and because the time-mean available enthalpy reservoir $A_{T M}$ is split into a large stratification component $A_{T M S}$ and a smaller but more active baroclinic component $A_{T M B}$. 
Figure 2 shows the domain- and time-averaged vertically integrated values of the energy reservoirs and energy fluxes for the month of December 2004. We see that the main flow of energy is as follows. The terms $G_{T M S}$ and $G_{T M B}$ generate time-mean available enthalpy $A_{T M S}$ and $A_{T M B}$ due to covariance of diabatic heating and temperature (although in this case $G_{T M B}$ is negligible and generation acts mostly upon $A_{T M S}$ ). Time-mean available enthalpy $A_{T M S}$ and $A_{T M B}$ are converted into time variability available enthalpy $A_{T V}$ through the action of eddy fluxes of heat acting upon timemean gradient of temperature (by the conversion terms $C_{A S}$ and $\left.C_{A B}\right)$. The covariance of vertical velocity and temperature deviations $\left(C_{T V}\right)$ then converts time variability available enthalpy $A_{T V}$ into time variability kinetic energy $K_{T V}$. Finally, the time variability kinetic energy $K_{T V}$ is partly destroyed by dissipative mechanisms $\left(D_{T V}\right)$, and to a lesser extent, baroclinically converted $\left(C_{K}\right)$ into time-mean kinetic energy $K_{T M}$, indicating that transient eddies contribute to maintaining the time-mean circulation. Hence, this regional energetics calculation shows that the most interesting transfers of energy occur between the four energy reservoirs $A_{T M B}, A_{T V}, K_{T M}$ and $K_{T V}$, with baroclinic conversions being the main process maintaining the atmospheric circulation over this mid-latitude limited-area domain.

It is interesting to compare the monthly averaged values in Fig. 2 with those presented in Fig. 1 for a 2-day period when a storm intensified rapidly over the studied domain. As expected, the baroclinic conversion terms are much larger during the rapid intensification period than the average over the month. For example, conversion term $c_{a}=13.3 \mathrm{~W} \mathrm{~m}^{-2}$ for the storm while $C_{A}=7.4 \mathrm{~W} \mathrm{~m}^{-2}$ for the monthly mean, and conversion term $c_{T V}=12.8 \mathrm{~W} \mathrm{~m}^{-2}$ for the storm while $C_{T V}=7.3 \mathrm{~W} \mathrm{~m}^{-2}$ for the monthly mean.

\section{Conclusions}

The aim of this paper was two-fold: (1) to develop a formalism suitable for the study of atmospheric energetics applicable over a limited region, and (2) to illustrate its application by studying the energy budgets of weather systems over a domain centred on the east of North America. The first goal was achieved through a relatively straightforward modification of the inter-member variability (IV) energy budget formulated by Nikiéma and Laprise (2013). The second goal was realised through the application of the formalism to a simulation of the fifth-generation Canadian Regional Climate Model (CRCM5) driven by reanalyses for the month of December 2004. A rapidly developing storm was first studied in detail to illustrate the dynamical and physical processes responsible for the evolution of individual weather systems. Then, the energy cycle for the whole month of December 2004 was computed to analyse the typical winter energetics over a North American domain.

For the study of atmospheric energetics at regional scale, available enthalpy is used rather than available potential energy, following Marquet (1996, Marquet 2003a, b) and Nikiéma and Laprise (2013). Over limited-area domains, the decomposition of atmospheric variables into their timemean state and time variability is better suited than into zonal-mean and deviations as is usually done for global energetics studies. The algebraic development of regional atmospheric energetics led to an extra reservoir in addition to the typical four of global energetics, due to the fact that the mass is not conserved over a limited region. It also proved convenient to decompose the time-mean state available enthalpy into a very large but rather inactive stratification component associated with the vertical variation of the temperature, and a smaller but more active baroclinic component associated with the horizontal variations of temperature. For the study of an individual weather system, only the time variability part of the energy cycle is relevant as a storm corresponds by definition to a deviation from the time-mean atmospheric state.

A low-pressure system that occurred in December 2004 over the domain was first studied in details. The storm started in the Atlantic Ocean, off the shore of Florida, and followed the coastline up to the Labrador Sea where it exited the domain through the northeast boundary. The pattern of time variability available enthalpy $a_{T V}$ and kinetic energy $k_{T V}$ clearly followed the temperature gradient anomaly created by the passage of the storm and the wind anomaly created by the circulation around it. The generation of energy $g_{T V}$ followed the front lines, and its dissipation $d_{T V}$, the maximum intensity of the wind. The conversion terms $\mathrm{c}_{\mathrm{a}}$ and $c_{T V}$ showed very similar fields and followed the storm track, transporting temperature to destroy the timemean temperature gradient.

For the monthly-mean energy cycle, available enthalpy of the time-mean state $A_{T M S}$ and $A_{T M B}$ were mainly generated by the covariance of diabatic heating and temperature $G_{T M S}$, and time variability kinetic energy $K_{T V}$ was mainly dissipated by the friction in the boundary layer $D_{T V}$. The available enthalpy was converted by $C_{A}$ from its time-mean state $A_{T M S}$ and $A_{T M B}$ into its time variability state $A_{T V}$ by eddy fluxes of heat acting to reduce the time-mean gradient of temperature. This time variability available enthalpy $A_{T V}$ was simultaneously converted by $C_{T V}$ into time variability kinetic energy $K_{T V}$ due to the covariance of time fluctuations of temperature and vertical velocity. These two important conversions corresponded to baroclinic processes and had their maximum amplitude along the storm track. Several boundary fluxes $(F$ and $H$ ) arose in the energy budget, acting either as sources or sinks of energy depending on the 
reservoir, but their values depended solely on the specific region and period studied. Hence, the regional energetics calculation indicated that baroclinic conversion was the main process maintaining the atmospheric circulation over this mid-latitude limited-area domain.

In the context of anticipated climate changes due to the continued release of greenhouse gases by anthropogenic activities, atmospheric energy budgets could be computed with future climate projections for the later part of this century. For instance, global energy budgets studies carried by Boer (1995) and Veiga and Ambrizzi (2013) indicate a reduction in the rate of generation of mean available potential energy and baroclinic conversions to eddy available potential energy and kinetic energy in a warming planet. Performing a comparison of current and future energy budgets over specific regions of the world could provide information on how the local energetics should be expected to change under global warming. Indeed, the location, intensity and frequency of individual storms would quite clearly be reflected in a storm's energetics and in the climatological energetics of that region.

Acknowledgments This research was funded by the Discovery Grant program of the Natural Sciences and Engineering Research Council of Canada (NSERC), the Climate Change and Atmospheric Research (CCAR) program of NSERC through a Grant to the Canadian Network for Regional Climate and Weather Processes (CNRCWP), Hydro-Québec and the OURANOS Consortium on Regional Climatology and Adaptation to Climate Change. The calculations were made possible by computing awards on the Compute Canada-Calcul Québec supercomputers. The authors thank Mr. Georges Huard, Mrs. Nadjet Labassi and Mrs. Katja Winger for maintaining an efficient and user-friendly local computing facility.

Open Access This article is distributed under the terms of the Creative Commons Attribution 4.0 International License (http://creativecommons.org/licenses/by/4.0/), which permits unrestricted use, distribution, and reproduction in any medium, provided you give appropriate credit to the original author(s) and the source, provide a link to the Creative Commons license, and indicate if changes were made.

\section{Appendix 1: development for $\partial a_{T V} / \partial t$}

Starting from NL13, Appendix 8, Eq. (109):

$$
\begin{aligned}
& \frac{\partial T^{\prime}}{\partial t}+\langle\vec{V}\rangle \cdot \vec{\nabla} T^{\prime}+\langle\omega\rangle \frac{\partial T^{\prime}}{\partial p}+\overrightarrow{V^{\prime}} \cdot \vec{\nabla}\langle T\rangle \\
& +\omega^{\prime} \frac{\partial\langle T\rangle}{\partial p}+\vec{\nabla} \cdot\left(\overrightarrow{V^{\prime}} T^{\prime}\right)+\frac{\partial\left(\omega^{\prime} T^{\prime}\right)}{\partial p}-\vec{\nabla} \cdot\left\langle\overrightarrow{V^{\prime}} T^{\prime}\right\rangle \\
& -\frac{\partial\left\langle\omega^{\prime} T^{\prime}\right\rangle}{\partial p}-\frac{R T_{r}}{c_{p} p} \omega^{\prime}-\frac{1}{c_{p}}\left(\frac{T_{r}}{T} Q-\left\langle\frac{T_{r}}{T} Q\right\rangle\right)=0
\end{aligned}
$$

We multiply Eq. (9) by $\frac{c_{p}}{T_{r}} T^{\prime}$ and add the expression $a_{T V} \cdot\langle\vec{\nabla} \cdot \vec{V}+\partial \omega / \partial p=0\rangle$ to it, using the definition $a_{T V} \equiv \frac{c_{p}}{2 T_{r}}\left(T^{2}\right):$

$$
\begin{aligned}
& \frac{\partial\left(a_{T V}\right)}{\partial t}+\vec{\nabla} \cdot\left(a_{T V}\langle\vec{V}\rangle\right)+\frac{\partial\left(a_{T V}\langle\omega\rangle\right)}{\partial p}+\left(\overrightarrow{V^{\prime}} \frac{T^{\prime}}{T_{r}}\right) \cdot \vec{\nabla}\left(c_{p}\langle T\rangle\right) \\
& +\left(\omega^{\prime} \frac{T^{\prime}}{T_{r}}\right) \frac{\partial\left(c_{p}\langle T\rangle\right)}{\partial p}+\frac{c_{p} T^{\prime}}{T_{r}} \vec{\nabla} \cdot\left(\overrightarrow{V^{\prime}} T^{\prime}\right)+\frac{c_{p} T^{\prime}}{T_{r}} \frac{\partial\left(\omega^{\prime} T^{\prime}\right)}{\partial p} \\
& -\frac{c_{p} T^{\prime}}{T_{r}} \vec{\nabla} \cdot\left\langle\overrightarrow{V^{\prime}} T^{\prime}\right\rangle-\frac{c_{p} T^{\prime}}{T_{r}} \frac{\partial\left\langle\omega^{\prime} T^{\prime}\right\rangle}{\partial p}-\omega^{\prime} \alpha^{\prime}-\frac{T^{\prime} Q}{T}-T^{\prime}\left\langle\frac{Q}{T}\right\rangle=0
\end{aligned}
$$

We rewrite the seventh and eleventh terms of Eq. (10):

$$
\begin{aligned}
& \frac{c_{p} T^{\prime}}{T_{r}} \vec{\nabla} \cdot\left(\overrightarrow{V^{\prime}} T^{\prime}\right)+\frac{c_{p} T^{\prime}}{T_{r}} \frac{\partial\left(\omega^{\prime} T^{\prime}\right)}{\partial p} \\
& \quad=\frac{c_{p}}{2 T_{r}} \vec{\nabla} \cdot\left(\overrightarrow{V^{\prime}} T^{\prime 2}\right)+\frac{c_{p} T^{\prime}}{T_{r}} \frac{\partial\left(\omega^{\prime} T^{\prime 2}\right)}{\partial p} \frac{T^{\prime} Q}{T} \\
& \quad=\frac{T^{\prime}\langle Q\rangle}{T}+\frac{T^{\prime} Q^{\prime}}{T}=\frac{T^{\prime}\langle Q\rangle}{T}+\frac{T_{r}}{T}\left(\frac{T^{\prime} Q^{\prime}}{T_{r}}\right)
\end{aligned}
$$

We insert Eq. (11) in Eq. (10) and rearrange the terms:

$$
\begin{aligned}
\frac{\partial\left(a_{T V}\right)}{\partial t}= & \frac{T_{r}}{T}\left(\frac{T^{\prime} Q^{\prime}}{T_{r}}\right)+\omega^{\prime} \alpha^{\prime}-\left(\overrightarrow{V^{\prime}} \frac{T^{\prime}}{T_{r}}\right) \cdot \vec{\nabla}\left(c_{p}\langle T\rangle\right) \\
& -\left(\omega^{\prime} \frac{T^{\prime}}{T_{r}}\right) \frac{\partial\left(c_{p}\langle T\rangle\right)}{\partial p}-\vec{\nabla} \cdot\left(a_{T V}\langle\vec{V}\rangle\right)-\frac{\partial\left(a_{T V}\langle\omega\rangle\right)}{\partial p} \\
& -\frac{c_{p}}{2 T_{r}} \vec{\nabla} \cdot\left(\overrightarrow{V^{\prime}} T^{\prime 2}\right)-\frac{c_{p}}{2 T_{r}} \frac{\partial\left(\omega^{\prime} T^{\prime 2}\right)}{\partial p}+\frac{c_{p} T^{\prime}}{T_{r}} \vec{\nabla} \cdot\left\langle\overrightarrow{V^{\prime}} T^{\prime}\right\rangle \\
& +\frac{c_{p} T^{\prime}}{T_{r}} \frac{\partial\left\langle\omega^{\prime} T^{\prime}\right\rangle}{\partial p}+T^{\prime}\left(\left\langle\frac{Q}{T}\right\rangle+\frac{\langle Q\rangle}{T}\right)
\end{aligned}
$$

We obtain the final form for the instantaneous time variability available enthalpy equation (Eq. 1) used in Sect. 2.1:

$\frac{\partial a_{T V}}{\partial t}=g_{T V}-c_{T V}+c_{a}-f_{a_{T V}}-h_{a_{T V}}-j_{a 1}-j_{a 2}$

Appendix 2: development for $\partial k_{T V} / \partial t$

Starting from the momentum equation:

$\frac{\partial \vec{V}}{\partial t}+\vec{V} \cdot \vec{\nabla} \vec{V}+\omega \frac{\partial \vec{V}}{\partial p}=-f \hat{k} \times \vec{V}-\vec{\nabla} \Phi+\vec{F}$

We apply the time-mean operator on Eq. (1):

$\frac{\partial\langle\vec{V}\rangle}{\partial t}+\langle\vec{V} \cdot \vec{\nabla} \vec{V}\rangle+\left\langle\omega \frac{\partial \vec{V}}{\partial p}\right\rangle=-f \hat{k} \times\langle\vec{V}\rangle-\vec{\nabla}\langle\Phi\rangle+\langle\vec{F}\rangle$

We compute the deviation from the time-mean state by subtracting Eq. (15) to Eq. (14): 


$$
\begin{aligned}
& \frac{\partial \overrightarrow{V^{\prime}}}{\partial t}+(\vec{V} \cdot \vec{\nabla} \vec{V}-\langle\vec{V} \cdot \vec{\nabla} \vec{V}\rangle)+\left(\omega \frac{\partial \vec{V}}{\partial p}-\left\langle\omega \frac{\partial \vec{V}}{\partial p}\right\rangle\right) \\
& =-f \hat{k} \times \overrightarrow{V^{\prime}}-\vec{\nabla} \Phi^{\prime}+\vec{F}^{\prime}
\end{aligned}
$$

We apply the following identity from Reynolds averaging rules to the second and third terms of Eq. (16):

$$
A B-\langle A B\rangle=A^{\prime}\langle B\rangle+B^{\prime}\langle A\rangle+A^{\prime} B^{\prime}-\left\langle A^{\prime} B^{\prime}\right\rangle
$$

$$
\begin{aligned}
(\vec{V} \cdot \vec{\nabla} \vec{V}-\langle\vec{V} \cdot \vec{\nabla} \vec{V}\rangle)= & \langle\vec{V}\rangle \cdot \vec{\nabla} \overrightarrow{V^{\prime}}+\overrightarrow{V^{\prime}} \cdot \vec{\nabla}\langle\vec{V}\rangle+\overrightarrow{V^{\prime}} \cdot \vec{\nabla} \overrightarrow{V^{\prime}} \\
& -\left\langle\overrightarrow{V^{\prime}} \cdot \vec{\nabla} \overrightarrow{V^{\prime}}\right\rangle\left(\omega \frac{\partial \vec{V}}{\partial p}-\left\langle\omega \frac{\partial \vec{V}}{\partial p}\right\rangle\right) \\
= & \langle\omega\rangle \frac{\partial \overrightarrow{V^{\prime}}}{\partial p}+\omega \frac{\partial\langle\vec{V}\rangle}{\partial p}+\omega \frac{\partial \overrightarrow{V^{\prime}}}{\partial p}-\left\langle\omega \frac{\partial \vec{V}^{\prime}}{\partial p}\right\rangle
\end{aligned}
$$

We insert Eq. (17) in Eq. (16):

$$
\begin{aligned}
& \frac{\partial \overrightarrow{V^{\prime}}}{\partial t}+\left(\langle\vec{V}\rangle \cdot \vec{\nabla} \overrightarrow{V^{\prime}}+\overrightarrow{V^{\prime}} \cdot \vec{\nabla}\langle\vec{V}\rangle\right. \\
& \left.\quad+\overrightarrow{V^{\prime}} \cdot \vec{\nabla} \overrightarrow{V^{\prime}}-\left\langle\overrightarrow{V^{\prime}} \cdot \vec{\nabla} \overrightarrow{V^{\prime}}\right\rangle\right) \\
& \quad+\left(\langle\omega\rangle \frac{\partial \overrightarrow{V^{\prime}}}{\partial p}+\omega^{\prime} \frac{\partial\langle\vec{V}\rangle}{\partial p}+\omega^{\prime} \frac{\partial \overrightarrow{V^{\prime}}}{\partial p}-\left\langle\omega^{\prime} \frac{\partial \overrightarrow{V^{\prime}}}{\partial p}\right\rangle\right) \\
& \quad=-f \hat{k} \times \overrightarrow{V^{\prime}}-\vec{\nabla} \Phi^{\prime}+\vec{F}^{\prime}
\end{aligned}
$$

We multiply Eq. (18) by $\vec{V}^{\prime}$ and use the definition $k_{T V}=\frac{1}{2}\left(\overrightarrow{V^{\prime}} \cdot \overrightarrow{V^{\prime}}\right)$

$$
\begin{aligned}
& \frac{\partial\left(k_{T V}\right)}{\partial t}+\langle\vec{V}\rangle \cdot \vec{\nabla} k_{T V}+\langle\omega\rangle \frac{\partial k_{T V}}{\partial p} \\
& +\left(\overrightarrow{V^{\prime}} \cdot \overrightarrow{V^{\prime}}\right) \vec{\nabla} \cdot\langle\vec{V}\rangle+\left(\overrightarrow{V^{\prime}} \cdot \overrightarrow{V^{\prime}}\right) \vec{V} \cdot \overrightarrow{V^{\prime}} \\
& -\overrightarrow{V^{\prime}}\left\langle\overrightarrow{V^{\prime}} \vec{\nabla} \cdot \overrightarrow{V^{\prime}}\right\rangle+\vec{V}^{\prime} \omega^{\prime} \frac{\partial\langle\vec{V}\rangle}{\partial p}+\overrightarrow{V^{\prime}} \omega^{\prime} \frac{\partial \overrightarrow{V^{\prime}}}{\partial p} \\
& -\overrightarrow{V^{\prime}}\left\langle\omega^{\prime} \frac{\partial \overrightarrow{V^{\prime}}}{\partial p}\right\rangle=-\overrightarrow{V^{\prime}} \cdot \vec{\nabla} \Phi^{\prime}+\overrightarrow{V^{\prime}} \cdot \overrightarrow{F^{\prime}}
\end{aligned}
$$

The fourth and tenth terms from Eq. (19) can be expressed as follows:

$$
\begin{array}{r}
\left(\overrightarrow{V^{\prime}} \cdot \overrightarrow{V^{\prime}}\right) \vec{\nabla} \cdot \overrightarrow{V^{\prime}}+\overrightarrow{V^{\prime}} \omega^{\prime} \frac{\partial \overrightarrow{V^{\prime}}}{\partial p}=\overrightarrow{V^{\prime}} \cdot \vec{\nabla} k_{T V}+\omega^{\prime} \frac{\partial k_{T V}}{\partial p} \\
-\overrightarrow{V^{\prime}} \cdot \vec{\nabla} \Phi^{\prime}=-\vec{\nabla} \cdot\left(\Phi^{\prime} \overrightarrow{V^{\prime}}\right)-\frac{\partial\left(\Phi^{\prime} \omega^{\prime}\right)}{\partial p}-\omega^{\prime} \alpha^{\prime}
\end{array}
$$

We insert Eq. (20) in Eq. (19):

$$
\begin{aligned}
& \frac{\partial\left(k_{T V}\right)}{\partial t}+\langle\vec{V}\rangle \cdot \vec{\nabla} k_{T V}+\langle\omega\rangle \frac{\partial k_{T V}}{\partial p}+\overrightarrow{V^{\prime}} \cdot \vec{\nabla} k_{T V}+\omega^{\prime} \frac{\partial k_{T V}}{\partial p} \\
& +\left(\overrightarrow{V^{\prime}} \cdot \overrightarrow{V^{\prime}}\right) \vec{\nabla} \cdot\langle\vec{V}\rangle-\overrightarrow{V^{\prime}}\left\langle\overrightarrow{V^{\prime}} \vec{\nabla} \cdot \overrightarrow{V^{\prime}}\right\rangle+\overrightarrow{V^{\prime}} \omega^{\prime} \frac{\partial\langle\vec{V}\rangle}{\partial p} \\
& -\overrightarrow{V^{\prime}}\left\langle\omega^{\prime} \frac{\partial \overrightarrow{V^{\prime}}}{\partial p}\right\rangle=-\vec{\nabla} \cdot\left(\Phi^{\prime} \overrightarrow{V^{\prime}}\right)-\frac{\partial\left(\Phi^{\prime} \omega^{\prime}\right)}{\partial p}-\omega^{\prime} \alpha^{\prime}+\overrightarrow{V^{\prime}} \cdot \overrightarrow{F^{\prime}}
\end{aligned}
$$

We take Eq. $(21)+k_{T V} \cdot\langle\vec{\nabla} \cdot \vec{V}+\partial \omega / \partial p=0\rangle$ to have the flux form of Eq. (21) and rearrange the terms:

$$
\begin{aligned}
\frac{\partial\left(k_{T V}\right)}{\partial t}= & -\omega^{\prime} \alpha^{\prime}-\overrightarrow{V^{\prime}} \cdot\left(\overrightarrow{V^{\prime}} \cdot \vec{\nabla}\right)\langle\vec{V}\rangle-\overrightarrow{V^{\prime}} \cdot\left(\omega^{\prime} \frac{\partial\langle\vec{V}\rangle}{\partial p}\right) \\
& +\overrightarrow{V^{\prime}} \cdot \overrightarrow{F^{\prime}}-\vec{\nabla} \cdot\left(k_{T V}\langle\vec{V}\rangle\right)-\frac{\partial\left(k_{T V}\langle\omega\rangle\right)}{\partial p} \\
& -\vec{\nabla} \cdot\left(k_{T V} \overrightarrow{V^{\prime}}\right)-\frac{\partial\left(k_{T V} \omega^{\prime}\right)}{\partial p}-\vec{\nabla} \cdot\left(\Phi^{\prime} \vec{V}^{\prime}\right)-\frac{\partial\left(\Phi^{\prime} \omega^{\prime}\right)}{\partial p} \\
& +\overrightarrow{V^{\prime}}\left(\left\langle\overrightarrow{V^{\prime}} \cdot \vec{\nabla} \overrightarrow{V^{\prime}}\right\rangle\right)+\overrightarrow{V^{\prime}}\left(\left\langle\omega^{\prime} \frac{\partial \overrightarrow{V^{\prime}}}{\partial p}\right\rangle\right)
\end{aligned}
$$

We obtain the final form for the instantaneous time variability kinetic energy equation (Eq. 2) used in Sect. 2.1:

$\frac{\partial k_{T V}}{\partial t}=c_{T V}+c_{k}-d_{T V}-f_{k_{T V}}-h_{k_{T V}}-j_{k 1}$

\section{References}

Boer GJ (1974) Zonal and eddy forms of the available potential energy equations in pressure coordinates. Tellus 27(2):433-442

Boer GJ (1995) Some dynamical consequences of greenhouse gas warming. Atmos Ocean 33(4):731-751

Dee DP, Uppala SM, Simmons AJ, Berrisford P, Poli P, Kobayashi S, Andrae U, Balmaseda MA, Balsamo G, Bauer P (2011) The ERA-Interim reanalysis: configuration and performance of the data assimilation system. Q J R Meteorol Soc 137:553-597. doi:10.1002/qj.828

Holton JR (2004) An introduction to dynamic meteorology. Academic Press, Cambridge, p 535

Lorenz EN (1955) Available potential energy and the maintenance of the general circulation. Tellus 7:157-167

Lorenz EN (1967) The nature and theory of the general circulation of the atmosphere. World Meteorological Organ 218 TP 115, p 161

Margules M (1905) Über die Energie der Stürme. In: Jahrbuch der Kaiserlich-Königliche Zentralanstalt für Meteorologie und Erdmagnetismus in Wien, NF, Bd. 42

Marquet P (1996) L'énergie utilisable de l'atmosphère. La Météorologie $8^{\mathrm{e}}$ série $14: 11-26$

Marquet P (2003a) The available-enthalpy cycle. I: introduction and basic equations. Q J R Meteorol Soc 129(593):2445-2466 
Marquet P (2003b) The available-enthalpy cycle. II: applications to idealized baroclinic waves. Q J R Meteorol Soc 129(593):2467-2494

Martynov A, Laprise R, Sushama L, Winger K, Šeparović L, Dugas B (2013) Reanalysis-driven climate simulation over CORDEX North America domain using the Canadian Regional Climate Model, version 5: Model performance evaluation. Clim Dyn 41(11-12):2973-3005

Newell RE, Kidson JW, Vincent DG, Boer GJ $(1972,1974)$ The general circulation of the tropical atmosphere and interactions with extratropical latitudes. MIT Press, Cambridge vol 1, p 320, and vol. 2, p 370

Nikiéma O, Laprise R (2013) An approximate energy cycle for intermember variability in ensemble simulations of a Regional Climate Model. Clim Dyn 46(1-2):831-852

Nikiéma O, Laprise R (2016) Energy cycle associated with intermember variability in a large ensemble of simulations with the Canadian RCM (CRCM5). Clim Dyn 46(1-2):651-670

Nikiéma O, Sommerfeld A, Laprise R, Rinke A, Dethloff K (2016) Chaos in Regional Climate Model simulations over an Arctic domain: comparison of inter-member variability in ensemble simulations of CRCM5 and HIRHAM5 models, Polarforschung (polar research) (in press)
Oort AH (1964a) On estimates of the atmospheric energy cycle. Mon Weather Rev 92:483-493

Oort AH (1964b) On the energetics of the mean and eddy circulations in the lower stratosphere. Tellus 26(3):309-327

Pearce RP (1978) On the concept of available potential energy. Q J R Meteorol Soc 104:737-755

Peixoto JP, Oort AH (1992) Physics of climate. American Institute of Physics, College Park, p 520

Smith PJ (1969a) A computational study of the energetics of a limited region of the atmosphere. Tellus 21(2):195-201

Smith PJ (1969b) On the contribution of a limited region to the global energy budget. Tellus 21(2):202-207

Spar J (1950) Synoptic studies of the potential energy in cyclones. J Meteorol 7:48-53

Tamura S (1905) Doctor Margules on the energy of storms. Mon Weather Rev 33:519-521

van Mieghem J (1973) Atmospheric energetics. Oxford Univ Press, Oxford, p 306

Veiga JAP, Ambrizzi T (2013) A global and hemispherical analysis of the Lorenz energetics based on the representative concentration pathways used in CMIP5. Adv Meteorol, ID 485047, p 13 